\title{
Analytical Investigation of Magnetohydrodynamic Non-Newtonian Type Casson Nanofluid Flow past a Porous Channel with Periodic Body Acceleration
}

\author{
N. Thamaraikannan $\mathbb{D}^{1},{ }^{1}$ S. Karthikeyan, ${ }^{1}$ Dinesh Kumar Chaudhary ${ }^{(D)}{ }^{2}$ \\ and Safak Kayikci ${ }^{3}{ }^{3}$ \\ ${ }^{1}$ Department of Mathematics, Erode Arts and Science College, Erode 638 009, Tamilnadu, India \\ ${ }^{2}$ Department of Physics, Amrit Campus, Tribhuvan University, Kathmandu, Nepal \\ ${ }^{3}$ Department of Computer Engineering, Bolu Abant Izzet Baysal University, Bolu, Turkey
}

Correspondence should be addressed to N. Thamaraikannan; kthamkar@gmail.com and Dinesh Kumar Chaudhary; din.2033@ gmail.com

Received 18 June 2021; Revised 27 August 2021; Accepted 28 August 2021; Published 17 September 2021

Academic Editor: Muhammad Imran Asjad

Copyright $\odot 2021 \mathrm{~N}$. Thamaraikannan et al. This is an open access article distributed under the Creative Commons Attribution License, which permits unrestricted use, distribution, and reproduction in any medium, provided the original work is properly cited.

\begin{abstract}
The consequence of periodic body acceleration and thermal radiation in the pulsating flow of MHD Casson nanofluid through a porous channel is addressed. A flow of the nanofluid injected through the lower plate is considered while sucked out through the upper plate with a similar velocity. The thermal radiation term is incorporated in the heat transfer equation. The governing equations corresponding to velocity and temperature are converted from partial differential equations to a system of ordinary differential equations by employing similarity variables. The perturbation technique is applied to solve the governing flow equations. The impact of diverse parameters on flow features is graphically analyzed. The result reveals that adding the nanoparticle has enhanced the velocity profile of the base fluid. Moreover, an increase in the periodic body acceleration results in enlarging velocity and temperature.
\end{abstract}

\section{Introduction}

The foremost principle of MHD is that forces are formed by the magnetic field, which stimulates a current through a moving conducting fluid. The importance of MHD in various areas such as astrophysics, biomedical research, and geophysics motivates us to investigate MHD flow. Concerning the shear strain-stress relationship, Casson fluid is a non-Newtonian fluid, which becomes firm when the yield stress is more significant than shear stress. However, it begins to deform when the yielded stress becomes less significant than shear stress. The MHD flow of Casson fluid in a porous channel is a field of dynamic research due to its applicability in industry and medical technology such as paper production, condensation, and blood flow in the human body. Due to its applications in heat storage beds and fossil fuels, heat and mass transfer through a porous medium have been developed into an exciting topic for the most recent decades. Tamoor et al. [1] focused on "MHD Casson fluid flow above a stretching cylinder." Reddy [2] examined the "MHD Casson fluid flow over an exponentially inclined permeable stretching surface with the influence of thermal radiation and chemical reaction on the flow of the MHD Casson fluid flow over an exponentially inclined permeable stretching surface."

Fluids with suspended nanoparticles have an extensive range of applications in the heat transfer process because an increment in thermal conductivity in fluids was noticed while mixing nanoparticles. This attempt was taken first by Choi [3] subsequently succeeded by Sandeep et al. [4] and Nisar et al. [5]. They suggested that "the nanoparticle-containing gold can be used to treat and trounce cancer." Next, Abolbashari et al. [6] 
analyzed "the mass and heat transfer characteristics of Casson nanofluid over a stretching surface." Finally, Tassaddiq et al. [7] analyzed "the Newtonian heating effects for the generalized Casson fluid MHD flow with porous effects." They found that velocity augments with escalating porosity and the Casson parameter control the fluid flow. Menni et al. [8, 9], Khan et al. [10], and Maouedj et al. [11] simulated the applicability of nanofluid in the solar channels.

Thermal radiation has a crucial role in many manufacturing processes. Irfan et al. [12] and Vellanki [13] investigated the radiation effect on Casson nanofluid and bio-nanofluid flow through a porous medium. MHD flow across porous channel between two parallel plates has vast application such as petrochemical engineering, polymer technology, food production technology, and biomedical research. Falede and Adensanya [14] explored "the oscillatory flow of MHD fluid through a porous channel." "MHD flow through a vertical channel with porous medium" was investigated by Dwivedi et al. [15]. "MHD nanofluid flow and heat transfer in rotating horizontal annulus were numerically simulated with thermal radiation" by Peng et al. [16]. The important visualization about the influence of magnetic field and radiation with assorted flow geometries is established in the literature [17-28].

The importance of the pulsating flow in a porous channel is well known, and it has many biological and industrial applications. Srinivas et al. [29] considered the MHD pulsating Casson fluid flow with chemical reaction and magnetic field through a porous channel. Kumar et al. [30] considered the "Joule heating and thermal radiation effects on the pulsatile MHD flow of Casson nanofluid through a vertical porous space." The study related to periodic body acceleration has enormous applications, particularly analyzing blood flow in the cardiovascular system. In many situations, such as driving in a vehicle, running, and jogging, the human body accelerates periodically (vibration). The sudden velocity change in the human body disturbs the blood flow, which causes most health problems. Vishali and Sarojamma [31] investigated the "flow of a fluid through a catheterized artery with the effect of a periodic body acceleration" in which they concluded that "the body acceleration enhanced velocity." Sulochana [32] discussed "the influence of body acceleration on an unsteady pulsatile flow of MHD couple stress fluid."

Over the years, nonlinear differential equations have been solved by approximate analytical methods such as the homotopy analysis method, weighted residual method, differential transformation methods, perturbation method, and so on. However, the non-perturbation approximate analytical methods have operational limitations that fussily tapered their performance domain, and they may sometimes lead to erroneous results while routinely implemented. Moreover, the conversion of the nonlinear equation and the evolution of corresponding recurrence equations using differential transformation methods are complex in some nonlinear systems. Consequently, the demand for reasonably simple, flexible, standard, and high precise total approximate analytical solutions is well acknowledged. One of such techniques applied for the quest is the perturbation method, which can solve nonlinear problems with high precision, while the perturbation method gives enhanced results for small perturbation parameters, further having a useful mathematical formulation with better accurateness, even for comparatively huge values of the perturbation parameter. Due to its unfussiness and high accuracy, the perturbation method was applied for solving various heat transfer and fluid mechanics problems [29-34].

To this extent, our literature review authenticates that there are no existing papers for MHD pulsating Casson nanofluid flow past a porous channel with periodic body acceleration effect. An examination was made for "pulsating Casson nanofluid flow through a porous channel with radiation" [35]. However, similar research for Casson nanofluid with periodic body acceleration effects was not investigated yet. Hence, we prolonged our effort to pulsative Casson nanofluid that passes through a porous channel between two parallel plates with periodic body acceleration and magnetic field impacts. The considered problem has massive application in manufacturing and biological processes. The perturbation technique was employed to solve the resulting similarity equations. The velocity and temperature fields are illustrated for several pertinent flow parameters.

\section{Mathematical Formulation}

Consider the influence of uniform traverse applied magnetic field on the pulsatile incompressible flow of a Casson nanofluid in a porous channel between two parallel plates, in which one plate is at rest and another plate moves in a parallel direction. The flow is driven by a pulsating pressure gradient [29].

$$
\frac{1}{\rho_{n f}} \frac{\partial p^{*}}{\partial x^{*}}=-A\left\{1+\varepsilon e^{i \omega t^{*}}\right\},
$$

where $\rho_{n f}$ is the "density of the nanofluid," $p^{*}$ is the "pressure," $\varepsilon \ll 1$ is suitably chosen positive quantity, $A$ is a constant, and $\omega$ is the "frequency." The physical flow system is noted in Figure 1.

We consider that $X^{*}$-axis is taken along the lower plate, and the perpendicular axis is noted as $Y^{*}$-axis. The lower plate is at rest, maintaining the temperature $T_{0}$. The upper plate is moving with the velocity $U_{w}^{*}=(A / \omega)\left\{1+\varepsilon e^{i \omega t^{*}}\right\}$ parallel to the $X^{*}$-axis and maintains the temperature $T_{1}\left(T_{0}<T_{1}\right)$. The nanofluid is injected from the lower plate into the porous channel with the velocity $v_{0}$, and it is sucked out from the porous medium via the upper plate with the similar velocity. "Uniform magnetic field" $\left(B_{0}\right)$ is imposed along the usual direction to the flow. The periodic body acceleration is assumed as $G^{*}=g_{0} \cos \psi$, where $\psi$ is the phase difference and $g_{0}$ is the amplitude of the body acceleration.

The Casson nanofluid rheological equation is stated as 


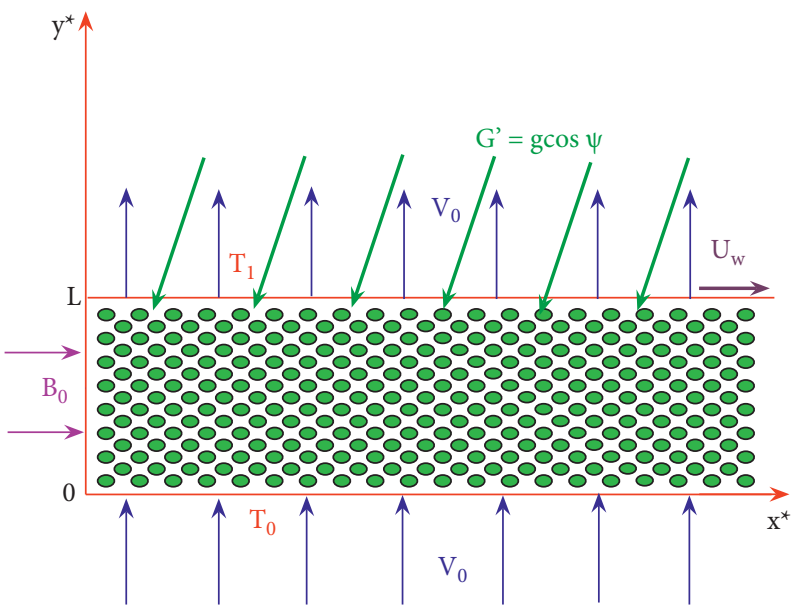

Figure 1: Physical model and coordinate system.

$$
T_{i j}= \begin{cases}\left(2 \mu_{B_{n f}}+\frac{p_{y}}{\sqrt{\left(\pi_{c} / 2\right)}}\right) e_{m n}, & \pi_{c}>\pi, \\ \left(2 \mu_{B_{n f}}+\frac{p_{y}}{\sqrt{(\pi / 2)}}\right) e_{m n}, & \pi_{c}<\pi,\end{cases}
$$

where " $\pi_{c}$ " is the critical value of " $\pi$ " based on the nonNewtonian model, $T_{m n}$ is the $(m, n)$-th stress tensor component, $\pi=e_{m n}$ with $e_{m n}$ being the $(m, n)$-th deformation rate, $\mu_{B_{n f}}$ is the plastic "dynamic viscosity" of the "nonNewtonian" nanofluid, and $p_{y}$ is the "yield stress" of the fluid.

The governing equations of the flow problem are stated as follows:

$$
\begin{aligned}
\frac{\partial u^{*}}{\partial x^{*}}= & 0 \\
\frac{\partial u^{*}}{\partial t^{*}}+v_{0} \frac{\partial u^{*}}{\partial y^{*}}= & -\frac{1}{\rho_{n f}} \frac{\partial p^{*}}{\partial x^{*}}+\frac{\mu_{n f}}{\rho_{n f}}\left(1+\frac{1}{\beta}\right) \frac{\partial^{2} u^{*}}{\partial y^{* 2}} \\
& -\frac{\sigma B_{0}^{2}}{\rho_{n f}} u^{*}-\frac{\mu_{n f} \Phi}{\rho_{n f} k} u^{*}+g_{0} \cos \psi \\
0= & -\frac{1}{\rho_{n f}} \frac{\partial p^{*}}{\partial y^{*}} \\
\frac{\partial T^{*}}{\partial t^{*}}+v_{0} \frac{\partial T^{*}}{\partial y^{*}}= & \frac{\kappa_{n f}}{\left(\rho C_{p}\right)_{n f}} \frac{\partial^{2} T^{*}}{\partial y^{* 2}}+\frac{\mu_{n f}}{\left(\rho C_{p}\right)_{n f}} \\
& \cdot\left(1+\frac{1}{\beta}\right)\left(\frac{\partial u^{*}}{\partial y^{*}}\right)^{2}+\frac{\sigma B 0^{2}}{\left(\rho C_{p}\right)_{n f}} u^{* 2} \\
& +\frac{4 \sigma^{*}}{3 K^{*}\left(\rho C_{p}\right)_{n f}} \frac{\partial^{2} T^{* 4}}{\partial y^{* 2}}+\frac{Q_{0}}{\left(\rho C_{p}\right)_{n f}}\left(T^{*}-T_{0}\right) .
\end{aligned}
$$

The boundary conditions for the present analysis are

$$
\begin{aligned}
& u^{*}=0, \\
& T^{*}=T_{0}, \quad \text { at } y^{*}=0, \\
& u^{*}=U_{w}^{*}=\frac{A}{\omega}\left\{1+\varepsilon e^{i \omega t^{*}}\right\}, \\
& T^{*}=T_{w}, \quad \text { at } y^{*}=L,
\end{aligned}
$$

where $Q_{0}$ is "heat source/sink parameter," $\beta$ is the "Casson parameter," $u^{*}$ is the "dimensional velocity in $X$-direction," $k$ and $\Phi$ are the "permeability and porosity of the porous medium," $\sigma$ is the "electrical conductivity" of the fluid, $C_{p_{n f}}$ is the "specific heat at constant pressure of nanofluid," $\mu_{n f}$ is the "dynamic viscosity" of the nanofluid, $\rho_{n f}$ is the "density" of the nanofluid, $\left(\rho C_{p}\right)_{n f}$ is the "heat capacity of the nanofluid," $v_{n f}$ is the "kinematic viscosity of the Casson nanofluid," $\kappa_{n f}$ is the "thermal conductivity" of the nanofluid, $K^{*}$ is "Rosseland mean absorption coefficient," and $\sigma^{*}$ is the "Stefan-Boltzmann constant" [16].

$$
\begin{aligned}
\rho_{n f} & =(1-\varphi) \rho_{f}+\varphi \rho_{n}, \\
\mu_{n f} & =\frac{\mu_{f}}{(1-\varphi)^{2.5}}, \\
\left(\rho C_{p}\right)_{n f} & =(1-\varphi)\left(\rho C_{p}\right)_{f}+\varphi\left(\rho C_{p}\right)_{n}, \\
\nu_{n f} & =\frac{\mu_{n f}}{\rho_{n f}}, \\
\frac{\kappa_{n f}}{\kappa_{f}} & =\frac{\left(2 \kappa_{f}+\kappa_{n}\right)-2 \varphi\left(\kappa_{f}-\kappa_{n}\right)}{\left(2 \kappa_{f}+\kappa_{n}\right)+\varphi\left(\kappa_{f}-\kappa_{n}\right)},
\end{aligned}
$$

where $\varphi$ is the nanoparticle volume fraction and the subscripts $n f, f$, and $n$ represent the nanofluid, base fluid, and nanoparticles correspondingly. The use of the above expression for $\left(\kappa_{n f} / \kappa_{f}\right)$ is restricted to spherical nanoparticles only. 
We assume that the temperature variation within the fluid flow can be expanded in a Taylor's series about $T_{0}$. By neglecting the higher-order terms, we obtain

$$
T^{* 4} \cong 4 T_{0}^{3} T^{*}-3 T_{0}^{4}
$$

We establish the dimensionless variables as follows:

$$
\begin{aligned}
x & =\frac{x^{*}}{L}, \\
y & =\frac{y^{*}}{L}, \\
t & =t^{*} \omega, \\
u & =\frac{u^{*} \omega}{A}, \\
p & =\frac{p^{*}}{\rho_{n f} A L}, \\
U_{w} & =\frac{\omega U_{w}^{*}}{A}, \\
\theta & =\frac{T^{*}-T_{0}}{T_{w}-T_{0}} .
\end{aligned}
$$

By applying equations (3), (5), (8), (9), and (10), in equations (4), (6), and (7),

$$
\begin{aligned}
& \frac{(1+(1 / \beta))}{(1-\varphi)^{2.5}\left(1-\varphi+\left(\varphi \rho_{n} / \rho_{f}\right)\right)} \frac{\partial^{2} u}{\partial y^{2}}-\operatorname{Re} \frac{\partial u}{\partial y} \\
& -\left(\frac{H a^{2}}{\left(1-\varphi+\left(\varphi \rho_{n} / \rho_{f}\right)\right)}+\frac{1}{\left(1-\varphi+\left(\varphi \rho_{n} / \rho_{f}\right)\right)(1-\varphi)^{2.5} \mathrm{Da}}\right) \\
& \cdot u=H^{2}\left(\frac{\partial u}{\partial t}+\frac{\partial p}{\partial x}\right)-G \cos \psi \\
& \frac{\left((4 / 3) \operatorname{Rd}+\left(k_{n f} / k_{f}\right)\right)}{\operatorname{Pr}} \frac{\partial^{2} \theta}{\partial y^{2}}-\left(1-\varphi+\frac{\varphi\left(\rho C_{p}\right)_{n}}{\left(\rho C_{p}\right)_{f}}\right) \operatorname{Re} \frac{\partial \theta}{\partial y} \\
& +Q \theta=H^{2}\left(1-\varphi+\frac{\varphi\left(\rho C_{p}\right)_{n}}{\left(\rho C_{p}\right)_{f}}\right) \frac{\partial \theta}{\partial t} \\
& -\operatorname{Ec}\left(\frac{(1+(1 / \beta))}{(1-\varphi)^{2.5}}\left(\frac{\partial u}{\partial y}\right)^{2}+\mathrm{Ha}^{2} u^{2}\right) .
\end{aligned}
$$

The new boundary conditions are

$$
\begin{aligned}
& u=0, \\
& \theta=0, \quad \text { at } y=0, \\
& u=U_{w}=\left\{1+\varepsilon e^{i t}\right\}, \\
& \theta=1, \quad \text { at } y=1,
\end{aligned}
$$

where $\mathrm{Da}=k / \Phi L^{2}$ is the "Darcy number of the porous media," $H=L \sqrt{\omega} / \sqrt{\nu_{f}}$ is the frequency parameter, $\mathrm{Ha}=$ $B_{0} L \sqrt{\sigma} / \sqrt{\mu_{f}}$ is the Hartmann number, $\operatorname{Re}=V_{0} L / \nu_{f}$ is the cross-flow Reynolds number, Ec $=\left((A / \omega)^{2} /\left(C_{p}\right)_{f}\left(T_{w}-\right.\right.$ $\left.\left.T_{0}\right)\right)$ is the Eckert number, $Q=\left(Q_{0} L^{2} /\left(\rho C_{p}\right)_{f} \nu_{f}\right)$ is the "heat source/sink parameter," $\mathrm{Rd}=\left(4 T_{0}^{3} \sigma^{*} / k_{f} K^{*}\right)$ is the radiation parameter, $\operatorname{Pr}=\left(\mu C_{p}\right)_{f} / k_{f}$ is the Prandtl number, and $G=$ $L^{2} \omega g_{0} / A \nu_{f}$ is the body acceleration parameter.

\section{Method of Solution}

The velocity $u$ and temperature $\theta$ can be supposed to have the form:

$$
\begin{aligned}
& u=u_{1}(y)+\varepsilon u_{2}(y) e^{i t}+\varepsilon^{2} u_{3}(y) e^{2 i t}, \\
& \theta=\theta_{1}(y)+\varepsilon \theta_{2}(y) e^{i t}+\varepsilon^{2} \theta_{3}(y)^{2 i t} .
\end{aligned}
$$

Now, substituting equations (1), (14), and (15) into equations (11)-(13) and then equating the coefficients of various powers of $\varepsilon$, we get

$$
\begin{gathered}
\alpha_{1} u_{1}^{l l}-\operatorname{Re} u_{1}^{l}-\alpha_{2} u_{1}=-H^{2}-G \cos \psi, \\
\alpha_{1} u_{2}^{l l}-\operatorname{Re} u_{2}^{l}-\alpha_{3} u_{2}=-H^{2}, \\
\alpha_{1} u_{3}^{l l}-\operatorname{Re} u_{3}^{l}-\alpha_{4} u_{3}=-H^{2}, \\
\beta_{1} \theta_{1}^{l l}-\beta_{2} \theta_{1}^{l}-Q \theta_{1}=-\beta_{3} u_{1}^{l 2}-\beta_{4} u_{1}^{2}, \\
\beta_{1} \theta_{2}^{l l}-\beta_{2} \theta_{2}^{l}-\beta_{5} \theta_{2}=-2 \beta_{3} u_{1}^{l} u_{2}^{l}-2 \beta_{4} u_{1} u_{2}, \\
\beta_{1} \theta_{3}^{l l}-\beta_{2} \theta_{3}^{l}-\beta_{6} \theta_{3}=-\beta_{3} u_{2}^{l 2}-\beta_{4} u_{2}^{2} .
\end{gathered}
$$

The new boundary conditions are

$$
\begin{aligned}
& u_{1}=0, \\
& u_{2}=0, \\
& u_{3}=0, \\
& \theta_{1}=0, \\
& \theta_{2}=0, \\
& \theta_{3}=0, \quad \text { at } y=0, \\
& u_{1}=1, \\
& u_{2}=1, \\
& u_{3}=1, \\
& \theta_{1}=1, \\
& \theta_{2}=0, \\
& \theta_{3}=0, \quad \text { at } y=1 .
\end{aligned}
$$

Solving the six ODEs (16)-(21) with the boundary conditions (22), we obtain 


$$
\begin{aligned}
u_{1}= & A_{1} e^{m_{1} y}+A_{2} e^{m_{2} y}+A_{3}, \\
u_{2}= & A_{4} e^{m_{3} y}+A_{5} e^{m_{4} y}+A_{6}, \\
u_{3}= & A_{7} e^{m_{5} y}+A_{8} e^{m_{6} y}+A_{9}, \\
\theta_{1}= & B_{8} e^{m_{7} y}+B_{7} e^{m_{8} y}+B_{1} e^{2 m_{1} y}+B_{2} e^{2 m_{2} y}+B_{3} e^{m_{1} y}+B_{4} e^{m_{2} y}+B_{5} e^{\left(m_{1}+m_{2}\right) y}+B_{6}, \\
\theta_{2}= & B_{18} e^{m_{9} y}+B_{19} e^{m_{10} y}+B_{9} e^{\left(m_{1}+m_{3}\right) y}+B_{10} e^{\left(m_{1}+m_{4}\right) y}+B_{11} e^{\left(m_{2}+m_{3}\right) y}+B_{12} e^{\left(m_{2}+m_{4}\right) y} \\
& +B_{13} e^{m_{1} y}+B_{14} e^{m_{2} y}+B_{15} e^{m_{3} y}+B_{16} e^{m_{4} y}+B_{17}, \\
\theta_{3}= & B_{37} e^{m_{11} y}+B_{36} e^{m_{12} y}+B_{20} e^{2 m_{3} y}+B_{21} e^{2 m_{4} y}+B_{22} e^{\left(m_{3}+m_{4}\right) y}+B_{23} e^{\left(m_{3}+m_{1}\right) y}+B_{24} e^{\left(m_{1}+m_{4}\right) y} \\
& +B_{25} e^{\left(m_{3}+m_{2}\right) y}+B_{26} e^{\left(m_{2}+m_{4}\right) y}+B_{27} e^{\left(m_{5}+m_{1}\right) y}+B_{28} e^{\left(m_{6}+m_{1}\right) y}+B_{29} e^{\left(m_{5}+m_{2}\right) y} \\
& +B_{30} e^{\left(m_{6}+m_{2}\right) y}+B_{31} e^{m_{3} y}+B_{32} e^{m_{4} y}+B_{33} e^{m_{5} y}+B_{34} e^{m_{6} y}+B_{35},
\end{aligned}
$$

where $m$ 's, $A$ 's, and $B$ 's are constants given in Appendix. by

The dimensionless Nusselt number at the plates is given

$$
\mathrm{Nu}=-\left(\frac{\partial \theta}{\partial y}\right)_{y=0,1}=-\left(\theta_{1}^{\prime}(y)+\varepsilon \theta_{2}^{\prime}(y) e^{i t}+\varepsilon^{2} \theta_{3}^{\prime}(y) e^{2 i t}\right)_{y=0,1} .
$$

\section{Results and Discussion}

The effect of diverse material parameters on the non-dimensional heat transfer and velocity is discussed in this section. The values of the Nusselt number are compared with previously available results obtained by Srinivas et al. [29] for the various assumptions of the Hartmann number, Reynolds number, radiation parameter, and heat source parameter to assess the validity of the current results, and the outcome is shown in Table 1 . Throughout the computation, the parameters are taken as $t=\pi / 4, \varepsilon=0.01, \mathrm{Re}=2, \phi=0.1$, Ha $=1, \beta=5, \mathrm{Da}=0.2, \mathrm{Ec}=0.2, Q=0.5, \mathrm{Rd}=3, \operatorname{Pr}=14, \psi=$ $60^{\circ}$, and $G=1$ unless otherwise stated. Figures $2(\mathrm{a})$ and $2(\mathrm{~b})$ represent the effect of nanoparticle volume fraction $(\varphi)$ and Hartmann number $(\mathrm{Ha})$ on the velocity distribution $u(y)$. It is noticed that $u(y)$ improves with an escalation in $\varphi$. Furthermore, the velocity is diminishing function of $\mathrm{Ha}$ since the applied magnetic field generates the Lorentz forces, which acts opposite to the flow direction. Figures $3(a)$ and 3(b) exhibit the effect of $H$ and $G$ on velocity distribution $u(y)$. It is noticed that $u(y)$ improves with an escalation in frequency parameter $(H)$ and body acceleration parameter $(G)$. Figure 4(a) elucidates the effect of $\mathrm{Da}$ on velocity distribution $u(y)$. It is evident that $u(y)$ improves with an escalation in Darcy number ( $\mathrm{Da}$ ) because $\mathrm{Da}$ is inversely proportional to the Darcy drag force. Figure 4(b) shows that an augment in Casson fluid parameter $(\beta)$ escalates velocity distribution $u(y)$.

Figures 5(a) and 5(b) show the influence of $(\mathrm{Ec})$ and $(G)$ on the temperature distribution $\theta(y)$. The observations conclude that the temperature distribution $\theta(y)$ upsurges with a rise in Ec. In addition, it is obvious that the escalating periodic body acceleration slightly improves the temperature.
Figures 6(a) and 6(b) displays persuade of (Re) and (Pr)on the temperature distribution $\theta(y)$. From Figure 6(a), the observations show that the temperature distribution $\theta(y)$ reduces with an upsurge in cross-flow Reynolds number. Figure 6(b) depicts that the temperature diminished for increasing values of Pr. The Prandtl number diminishes the thermal boundary layer thickness. In addition, it controls the thickness of the thermal boundary layer. Fluids with lower Prandtl number have higher thermal conductivity so that the heat can diffuse from the plate faster than higher Prandtl number fluids [29]. Figures 7(a) and 7 (b) reveal the influence of $Q$ and $\operatorname{Rd}$ on $\theta(y)$. Figure 7(a) depicts that the temperature distribution boosts up with a rise in the heat source/sink parameter $(Q)$. From Figure $7(\mathrm{~b})$, one can observe a rise in $\theta(y)$ with intensification in $\mathrm{Rd}$. This is in agreement with the physical reality that the thermal boundary layer thickness enhances with growing Rd.

The effect of Eckert number (Ec), Darcy number (Da), body acceleration parameter $(G)$, radiation parameter $(\mathrm{Rd})$, the nanoparticle volume fraction $(\varphi)$, Hartmann number $(\mathrm{Ha})$, heat source/sink parameter $(Q)$, and Prandtl number $(\mathrm{Pr})$ on unsteady temperature $\theta_{t}(y)$ is shown in Figures 8-11. From Figure 8(a), it is observed that the enhancement in Eckert number (Ec) produces an increasing tendency in unsteady temperature $\theta_{t}(y)$. Figure $8(\mathrm{~b})$ depicts that the unsteady temperature $\theta_{t}(y)$ is oscillating with increasing Darcy number (Da). From Figure 9(a), it is observed that the increase in body acceleration parameter $(G)$ produces an increase in unsteady temperature $\theta_{t}(y)$. Figure 9(b) shows that the unsteady temperature $\theta_{t}(y)$ is oscillating with increasing Hartmann number ( $\mathrm{Ha}$ ).

Figure 10(a) shows that the amplification in the nanoparticle volume fraction $(\varphi)$ produces an insurgence in unsteady temperature $\theta_{t}(y)$ at the lower half of the channel; however, it begins osculating near the upper wall. From Figure 10(b), it is evident that the increase in Pr escalates the unsteady temperature.

From Figure 11(a), the influence of $Q$ is minimal in the lower part of the channel. However, the unsteady temperature slightly decreases on the upper part of the channel for the increasing values of $Q$. From Figure 11(b), it is noticed 
TABle 1: Comparison of Nusselt number for Newtonian and non-Newtonian fluids with Srinivas et al. [29] for the limiting case of $\varphi=0, G=0, \varepsilon=0.01, H=3, \beta=2, \mathrm{Ec}=1, \mathrm{Da}=0.1, \operatorname{Pr}=21$, and $t=\pi / 4$.

\begin{tabular}{|c|c|c|c|c|c|c|c|c|c|}
\hline \multirow{3}{*}{ Parameter } & \multirow{3}{*}{ Values } & \multicolumn{4}{|c|}{$\mathrm{Nu}=-(\partial \theta / \partial y)_{y=0}$} & \multicolumn{4}{|c|}{$\mathrm{Nu}=-(\partial \theta / \partial y)_{y=1}$} \\
\hline & & \multicolumn{2}{|c|}{ Newtonian } & \multicolumn{2}{|c|}{ Non-Newtonian } & \multicolumn{2}{|c|}{ Newtonian } & \multicolumn{2}{|c|}{ Non-Newtonian } \\
\hline & & [29] & Present & [29] & Present & [29] & Present & [29] & Present \\
\hline & 0 & -2.7033 & -2.7033 & -2.6361 & -2.6361 & 3.1771 & 3.1771 & 2.7457 & 2.7457 \\
\hline \multirow{3}{*}{$\mathrm{Ha}$} & 2 & -2.2726 & -2.2726 & -2.2307 & -2.2307 & 3.5754 & 3.5754 & 2.7261 & 2.7261 \\
\hline & 4 & -1.5615 & -1.5615 & -1.5380 & -1.5380 & 2.6740 & 2.6740 & 1.8116 & 1.8116 \\
\hline & 0 & -7.6829 & -7.6829 & -7.1678 & -7.1678 & 6.1846 & 6.1846 & 5.6695 & 5.6695 \\
\hline \multirow[t]{3}{*}{$\operatorname{Re}$} & 1 & -2.2726 & -2.2726 & -2.2307 & -2.2307 & 3.5754 & 3.5754 & 2.7261 & 2.7261 \\
\hline & 2 & -1.1585 & -1.1585 & -1.2067 & -1.2067 & -1.9577 & -1.9577 & -2.8056 & -2.8056 \\
\hline & 0 & -2.5506 & -2.5506 & -2.4868 & -2.4868 & 1.6763 & 1.6763 & 1.2142 & 1.2142 \\
\hline \multirow[t]{3}{*}{ Q } & 0.5 & -2.6712 & -2.6712 & -2.6064 & -2.6064 & 3.2306 & 3.2306 & 2.7556 & 2.7556 \\
\hline & 1 & -2.8333 & -2.8333 & -2.7671 & -2.7671 & 5.4356 & 5.4356 & 4.9425 & 4.9425 \\
\hline & 0 & -4.2702 & -4.2702 & -4.1034 & -4.1034 & 19.2777 & 19.2777 & 17.4188 & 17.4188 \\
\hline \multirow[t]{2}{*}{$\mathrm{Rd}$} & 1 & -3.3850 & -3.3850 & -3.2904 & -3.2904 & 6.2590 & 6.2590 & 5.4984 & 5.4984 \\
\hline & 2 & -2.8333 & -2.8333 & -2.7671 & -2.7671 & 3.2303 & 3.2303 & 2.7556 & 2.7556 \\
\hline
\end{tabular}

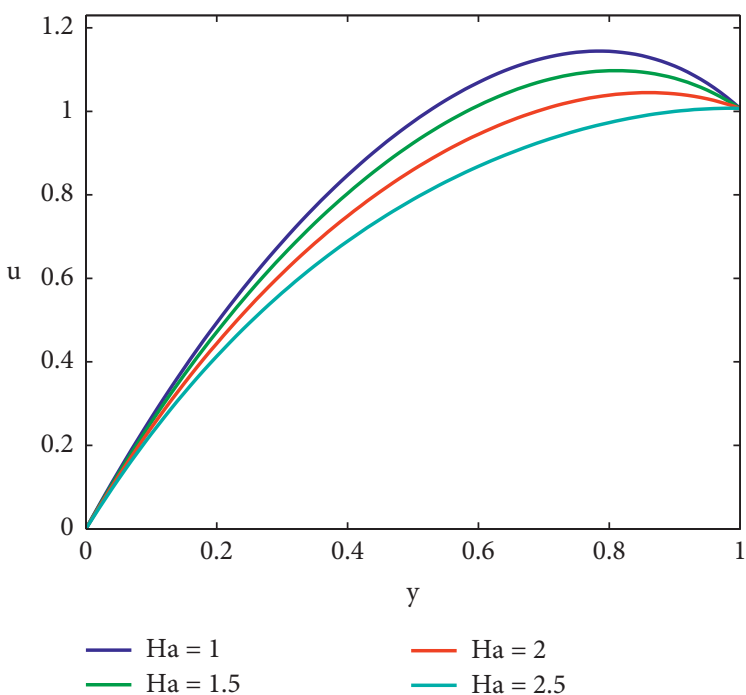

(a)

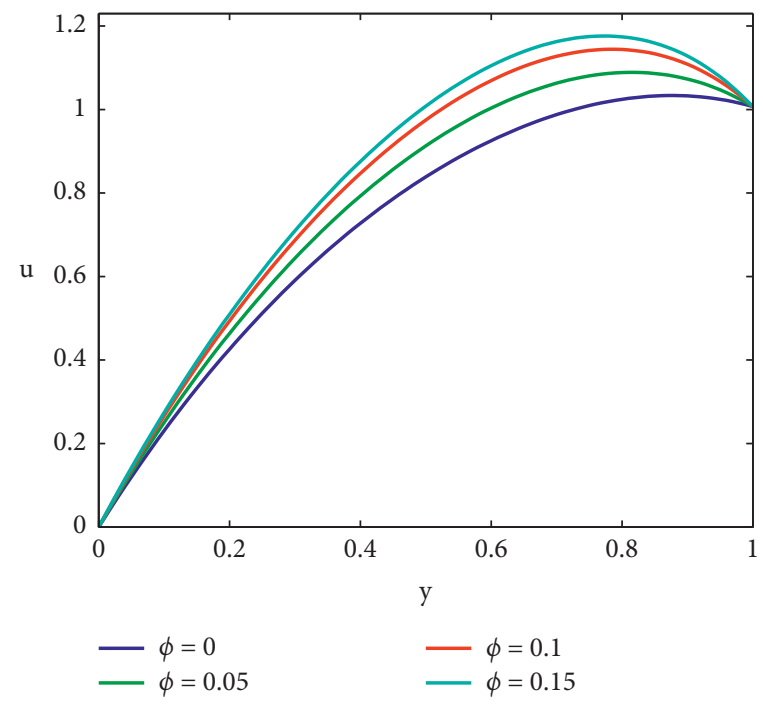

(b)

FIgURE 2: (a) Effect of Ha on the "velocity distribution" when $\varphi=0.1$. (b) Effect of $\varphi$ on the "velocity distribution" when Ha $=1$.

that the increase in radiation parameter $(\mathrm{Rd})$ produces a diminishing tendency in unsteady temperature.

Figure 12(a) shows the influence of the nanoparticle volume fraction $(\varphi)$ on the Nusselt number $(\mathrm{Nu})$ distribution. It is observed that a given rise in $\varphi$ results in the decrease of Nusselt number $(\mathrm{Nu})$ at the lower plate, although it increases at the upper plate. Figure 12(b) shows the influence of the body acceleration parameter $(G)$ on the Nusselt number $(\mathrm{Nu})$ distribution. It is observed that a given increase in body acceleration at the lower plate results in the decrease of Nusselt number $(\mathrm{Nu})$, while it increases at the upper plate.

Figure 13 shows the influence of $\mathrm{Rd}$ on the Nusselt number $(\mathrm{Nu})$ distribution. It is observed that a given increase in the $\mathrm{Rd}$ results in the decline of the Nusselt number $(\mathrm{Nu})$ at the upper plate, while it increases 

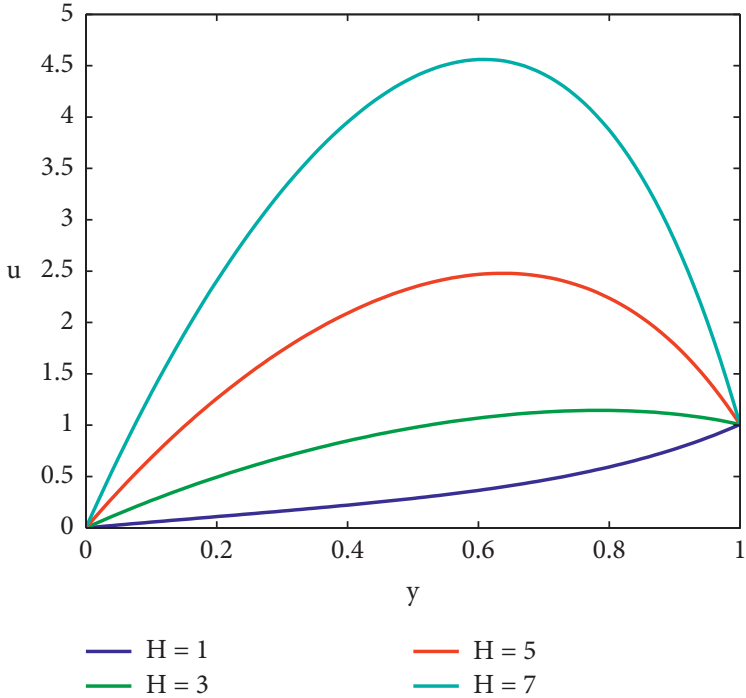

(a)

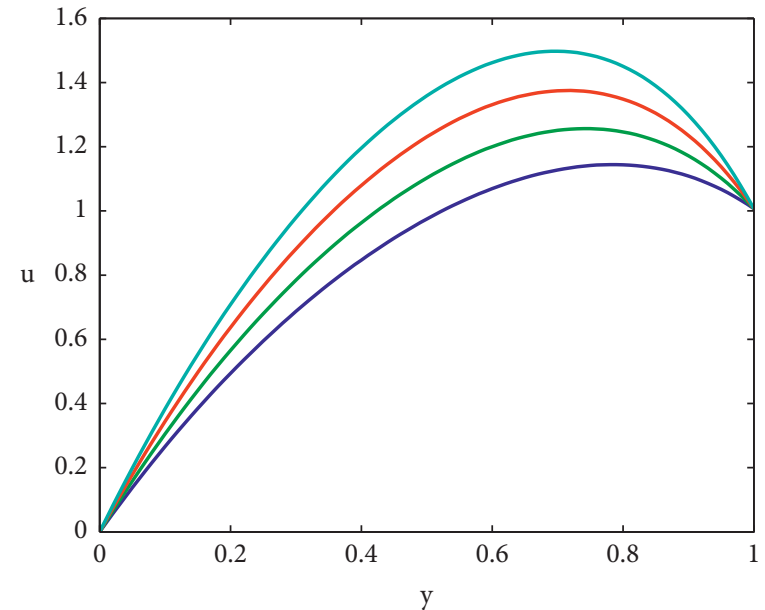

$-\mathrm{G}=1$

$-\mathrm{G}=7$

$-\mathrm{G}=10$

(b)

Figure 3: (a) Effect of $H$ on the "velocity distribution" when $G=1$. (b) Effect of $G$ on the velocity distribution when $H=5$.
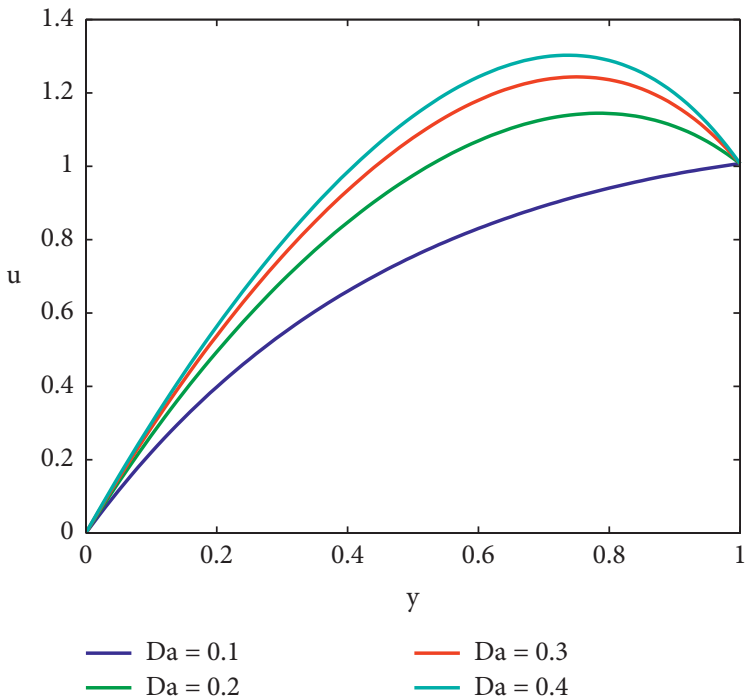

(a)

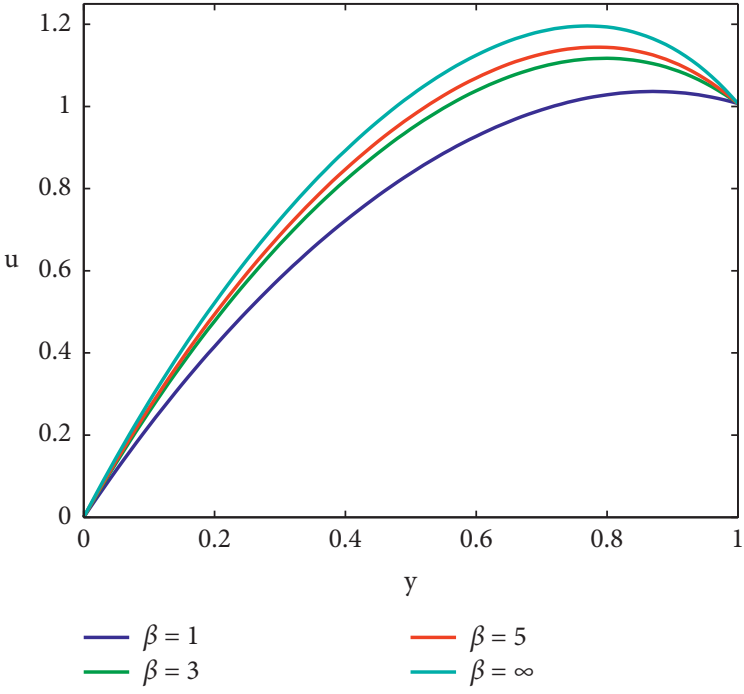

(b)

FIgUre 4: (a) Effect of Da on the "velocity distribution" when $\beta=5$. (b) Effect of $\beta$ on the "velocity distribution" when Da $=0.2$. 


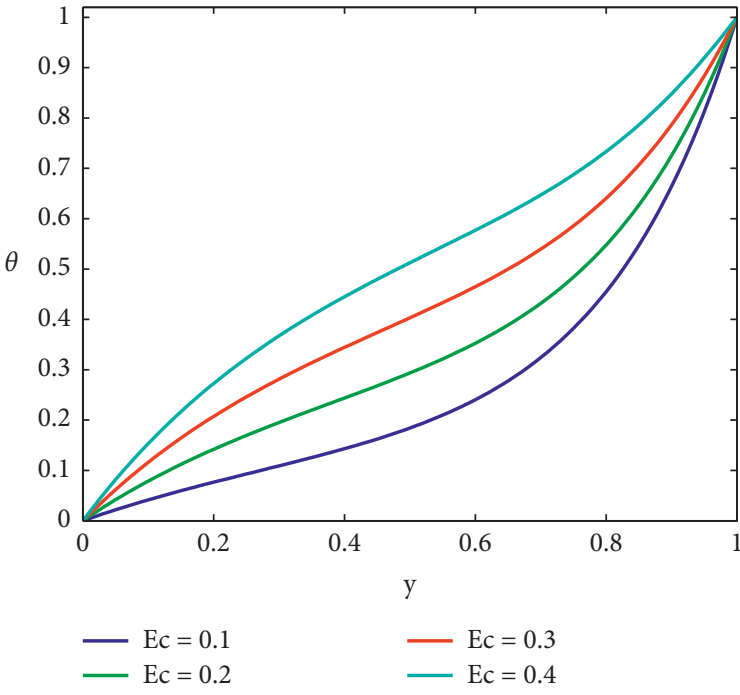

(a)

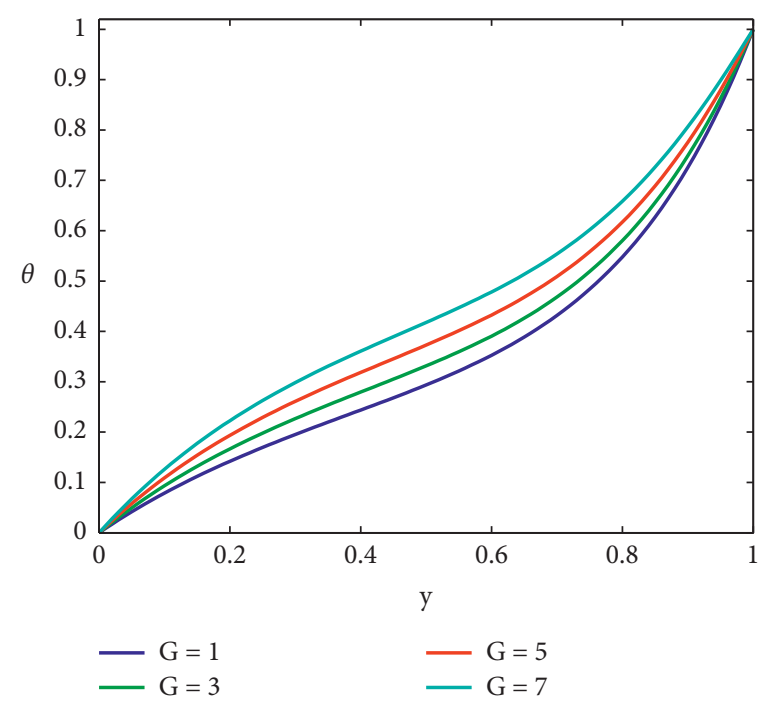

(b)

Figure 5: (a) Effect of Ec on the "temperature distribution" when $G=1$. (b) Effect of $G$ on the "temperature distribution" when Ec $=0.2$.

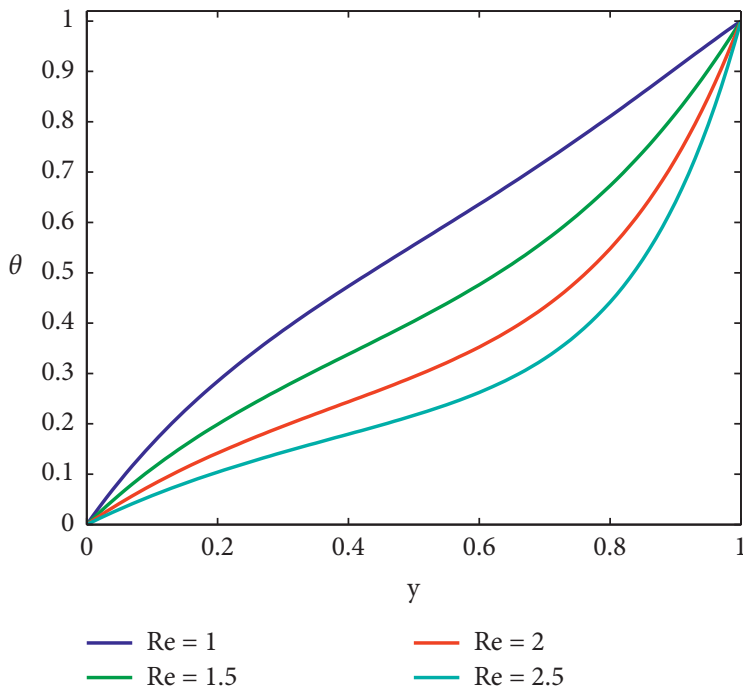

(a)

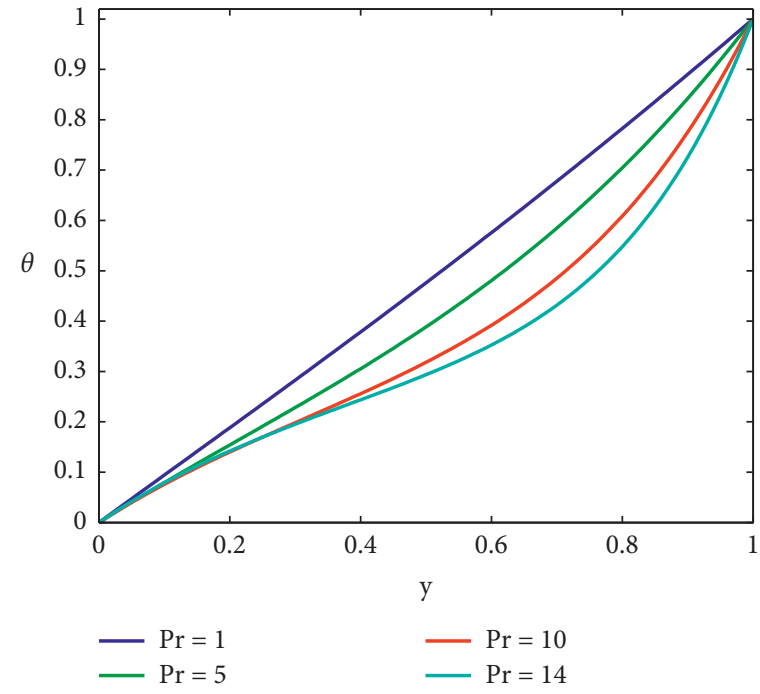

(b)

Figure 6: (a) Effect of Re on the "temperature distribution" when $\operatorname{Pr}=14$. (b) Effect of Pr on the temperature distribution when $\operatorname{Re}=2$. 


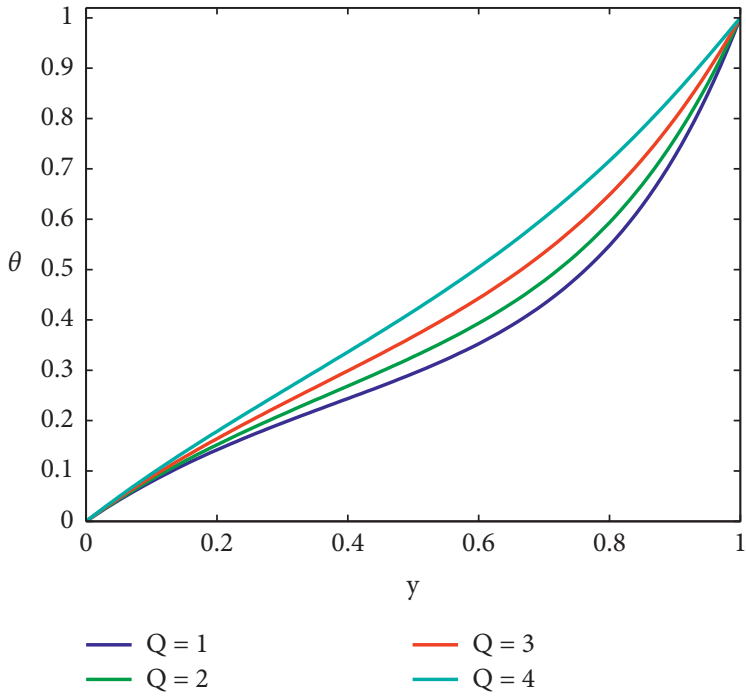

(a)

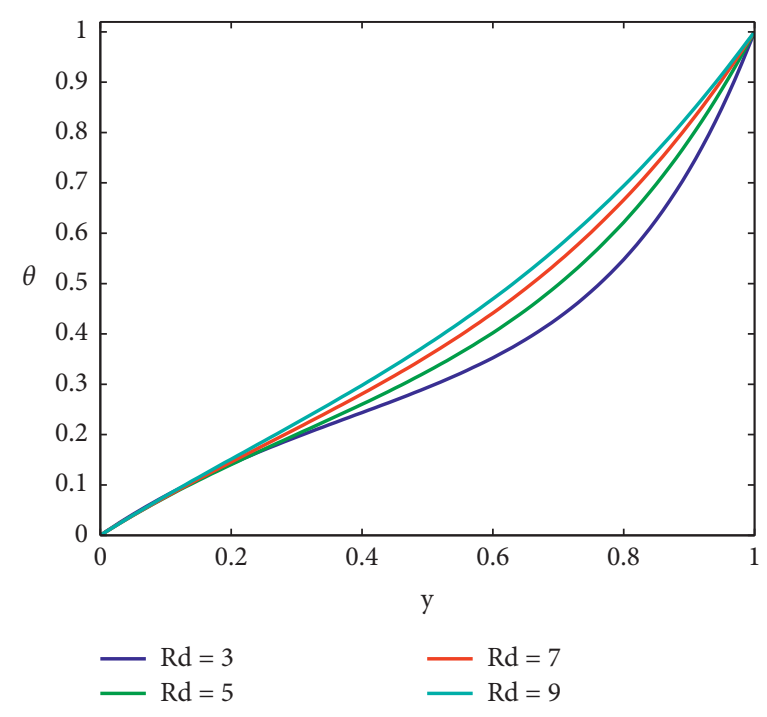

(b)

Figure 7: (a) Effect of $Q$ on the "temperature distribution" when $\mathrm{Rd}=3$. (b) Effect of $\mathrm{Rd}$ on the "temperature distribution" when $Q=0.5$.

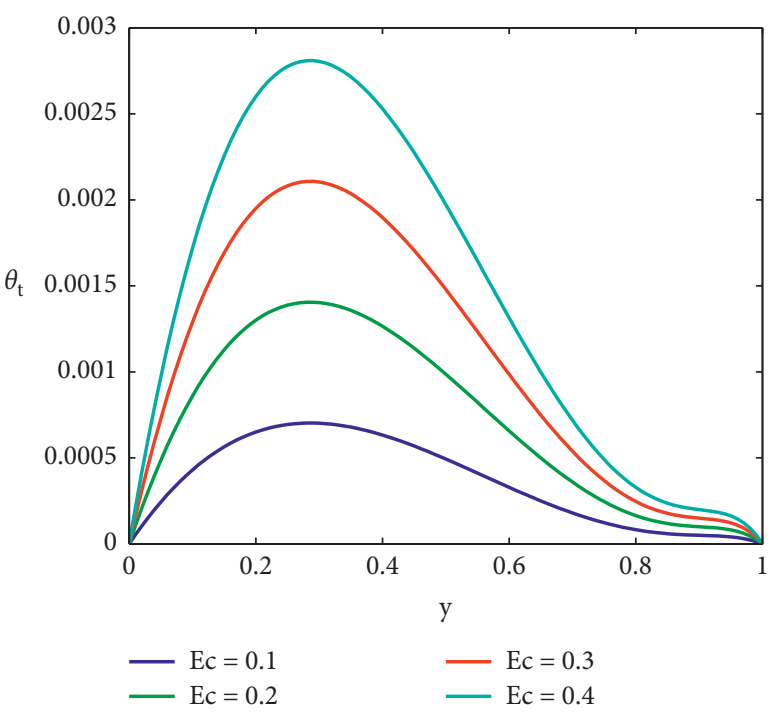

(a)

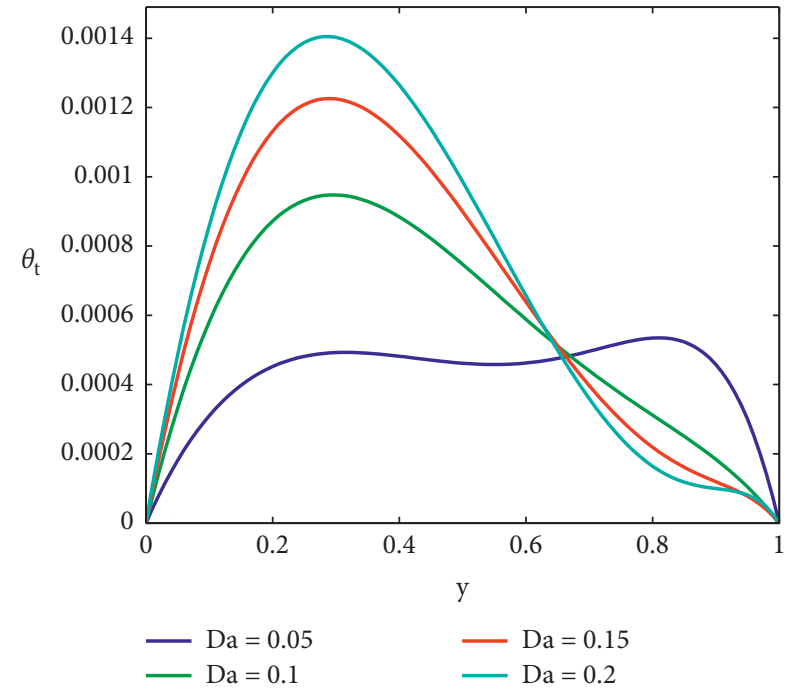

(b)

FiguRE 8: (a) Effect of Ec on "unsteady temperature distribution" for Da = 0.2. (b) Effect of Da on "unsteady temperature distribution" for $\mathrm{Ec}=0.2$. 


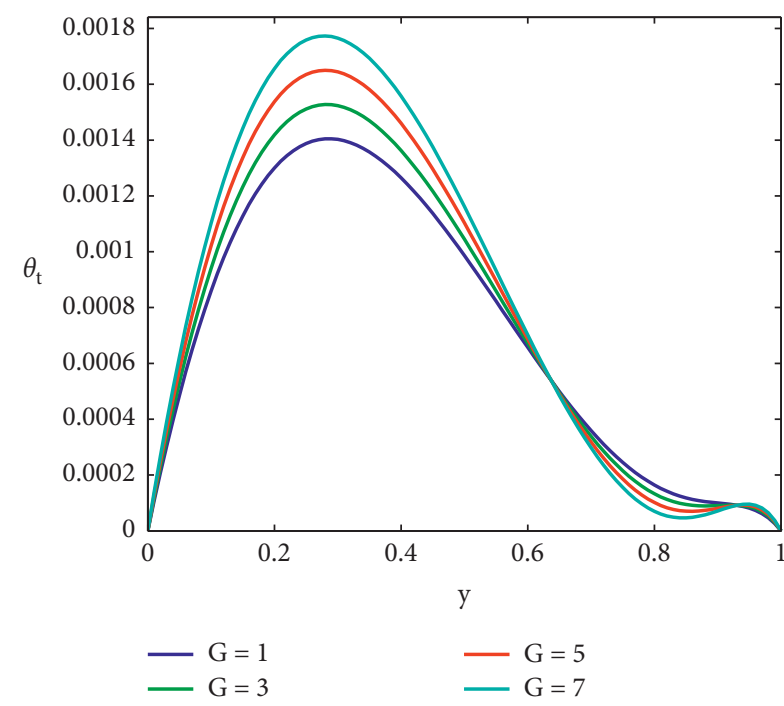

(a)
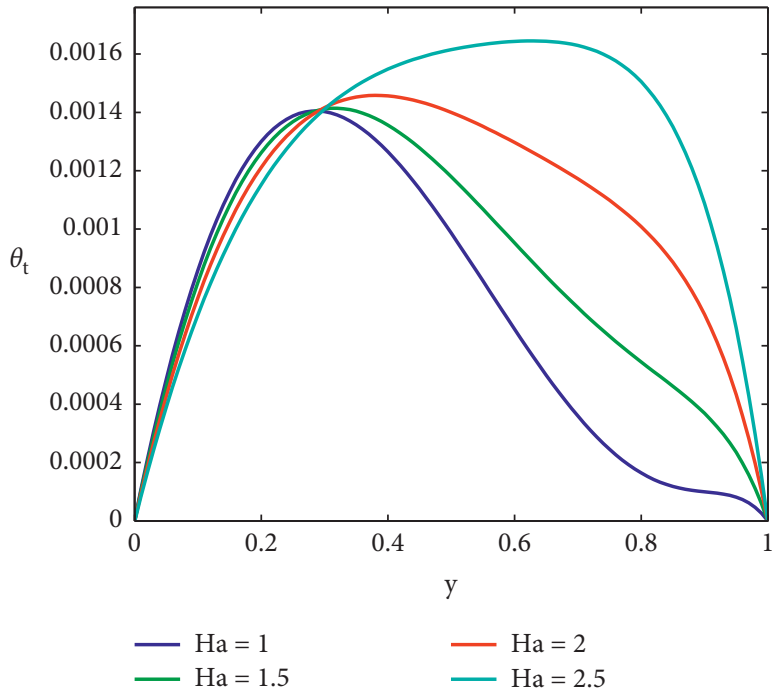

(b)

Figure 9: (a) Effect of $G$ on unsteady "temperature distribution" when $\mathrm{Ha}=1$. (b) Effect of Ha on "unsteady temperature distribution" for $G=1$.

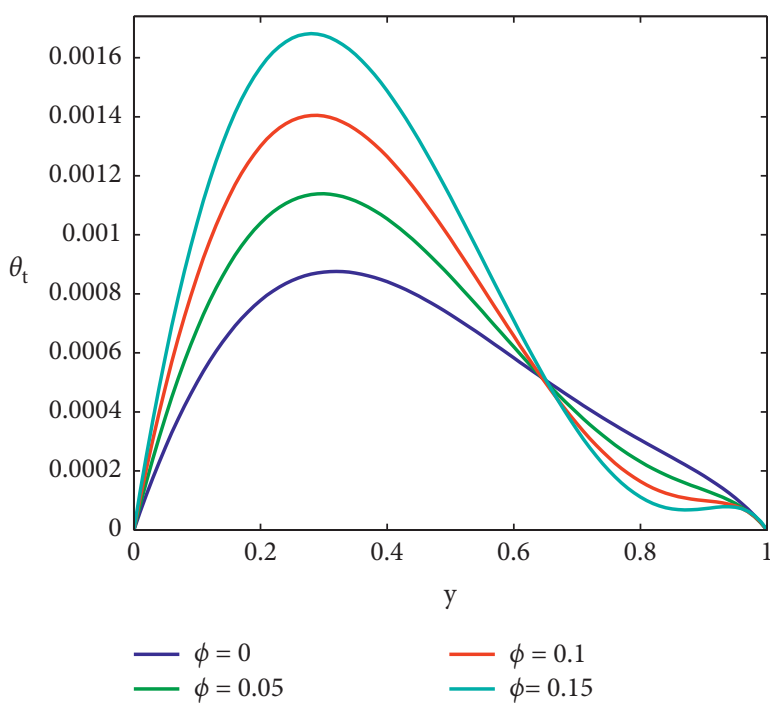

(a)

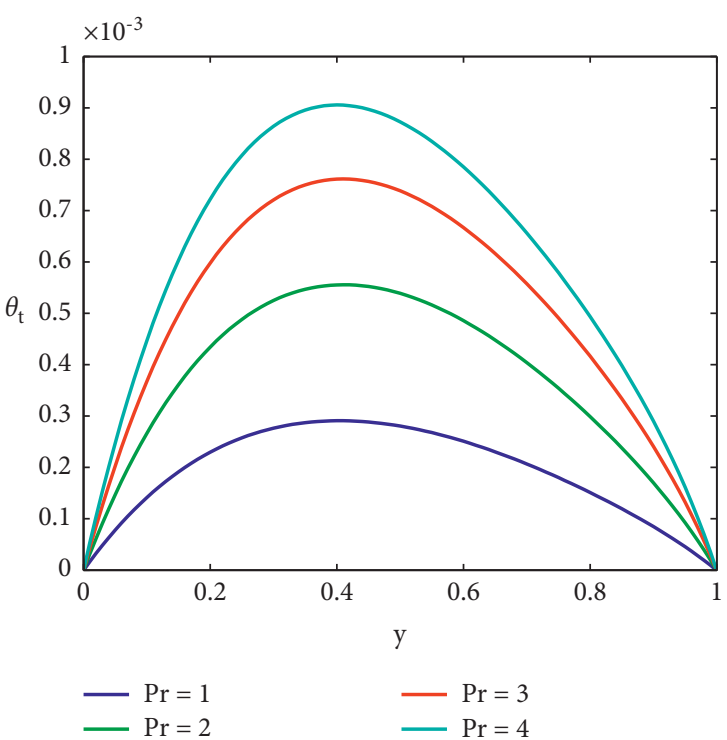

(b)

Figure 10: (a) Effect of $\varphi$ on "unsteady temperature distribution" when $\operatorname{Pr}=14$. (b) Effect of $\operatorname{Pr}$ on "unsteady temperature distribution" for $\varphi=0.1$. 


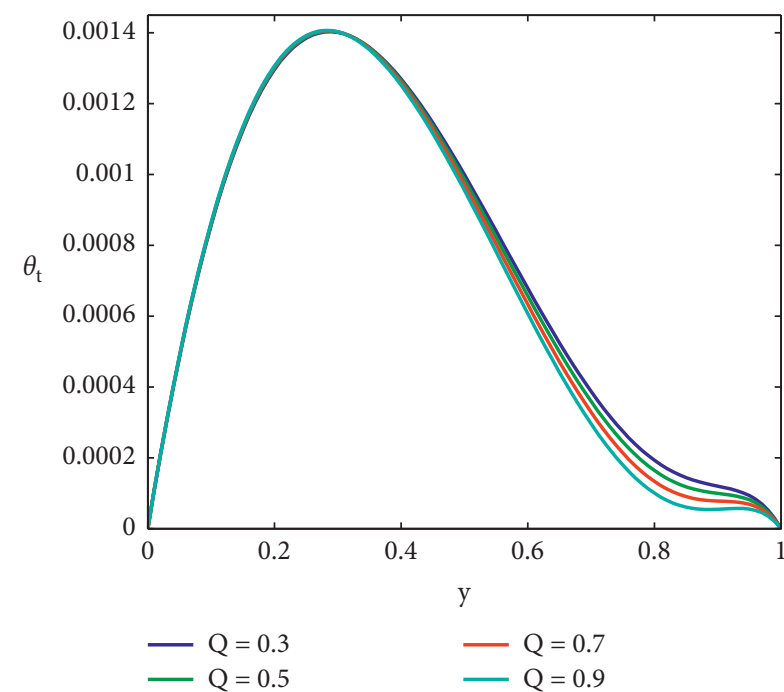

(a)

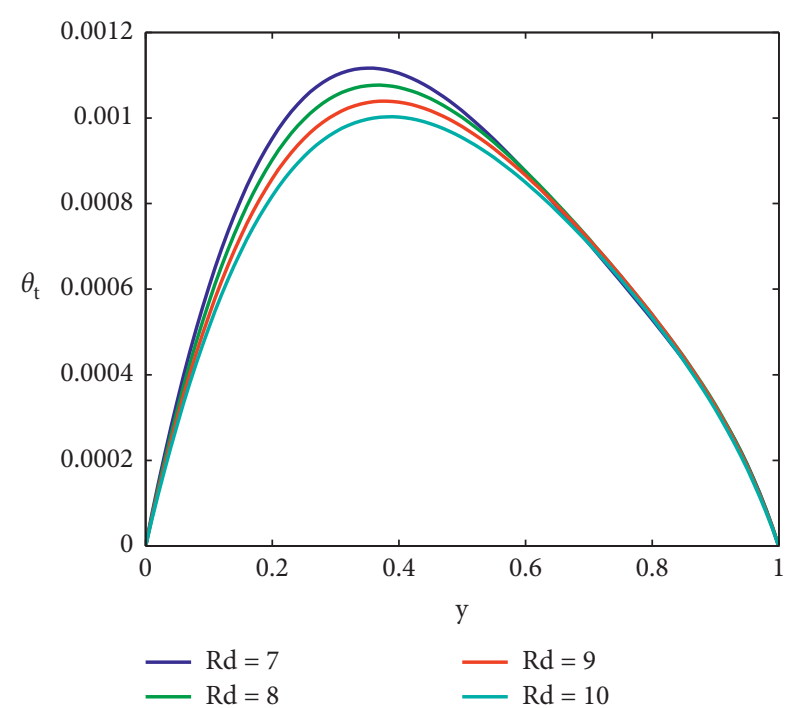

(b)

Figure 11: (a) Effect of Q on "unsteady temperature distribution" when Rd = 3. (b) Effect of Rd on "unsteady temperature distribution" for $Q=0.5$.

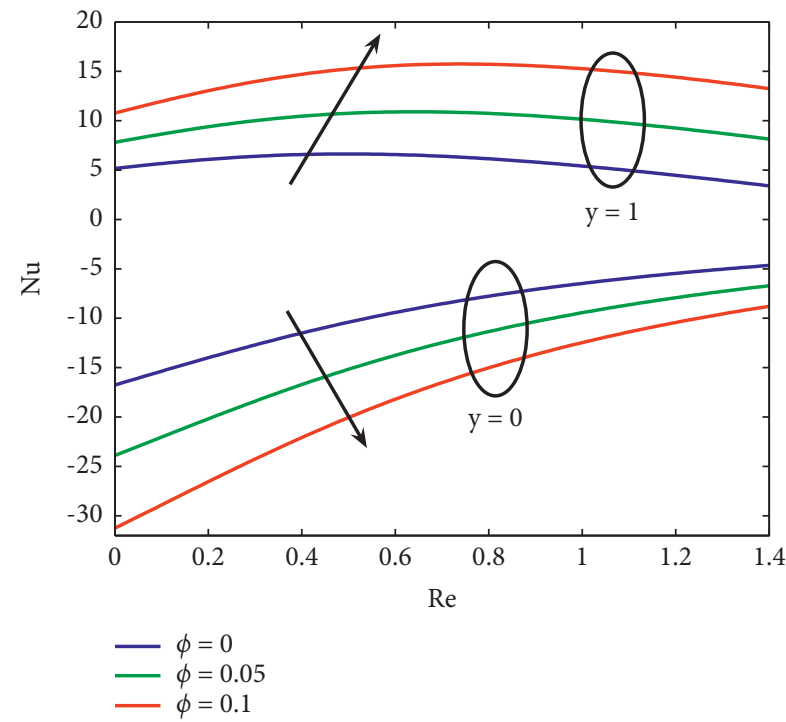

(a)

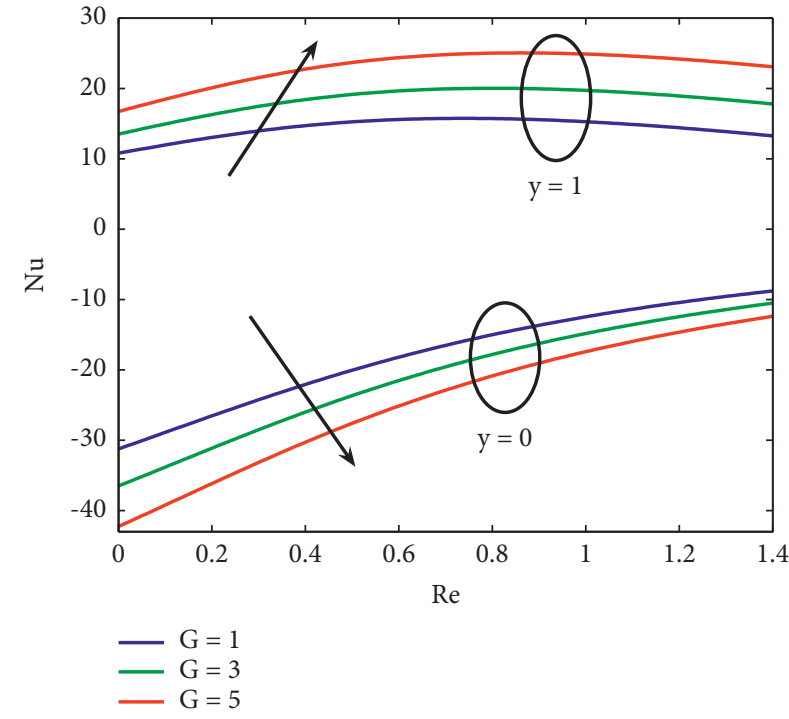

(b)

Figure 12: (a) Effect of $\varphi$ on "Nusselt number distribution" when $G=1, \mathrm{Ec}=1, \mathrm{Ha}=0.5, \mathrm{Rd}=2$, and $\operatorname{Pr}=21$. (b) Effect of G on "Nusselt number distribution" for $\varphi=0.1, \mathrm{Ec}=1, \mathrm{Ha}=0.5, \mathrm{Rd}=2$, and $\operatorname{Pr}=21$.

at the lower plate. The greatest rate of change for $\mathrm{Nu}$ in both the plates was seen near the null values of $\mathrm{Rd}$ and Re.

Figure 14 illustrates the variation of the unsteady Nusselt number $\left(\mathrm{Nu}_{t}\right)$ for Re with time variation in the upper plate. It is observed that the unsteady Nusselt number upsurges with increasing Re. Thus, the unsteady Nusselt number distribution is a periodic function of " $t$ " with period $2 \pi$.

Table 2 shows the variations of the Nusselt number $(\mathrm{Nu})$ for the Casson nanofluid with and without body acceleration. The periodic body acceleration is taken as the body acceleration parameter $G=1$. From this table, 


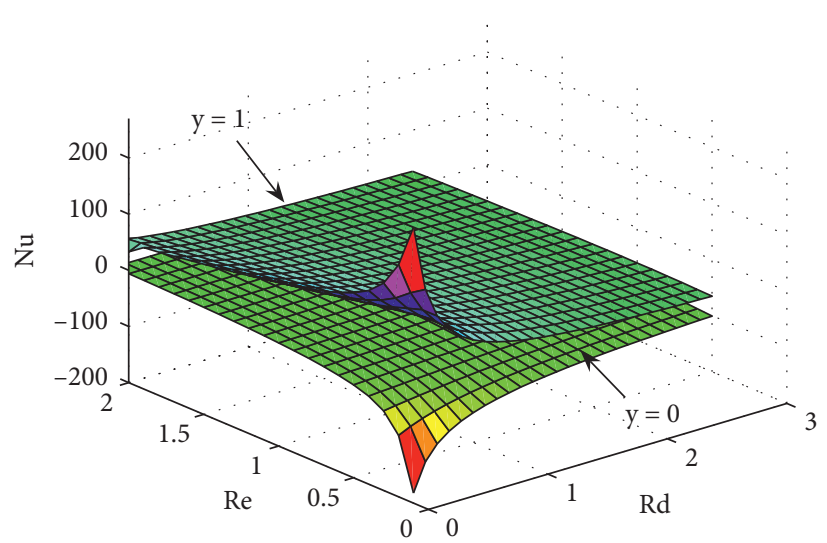

FIgURE 13: Effect of Rd and Re on "the Nusselt number distribution" when $\mathrm{Ec}=1, \mathrm{Ha}=0.5$, and $\operatorname{Pr}=21$.

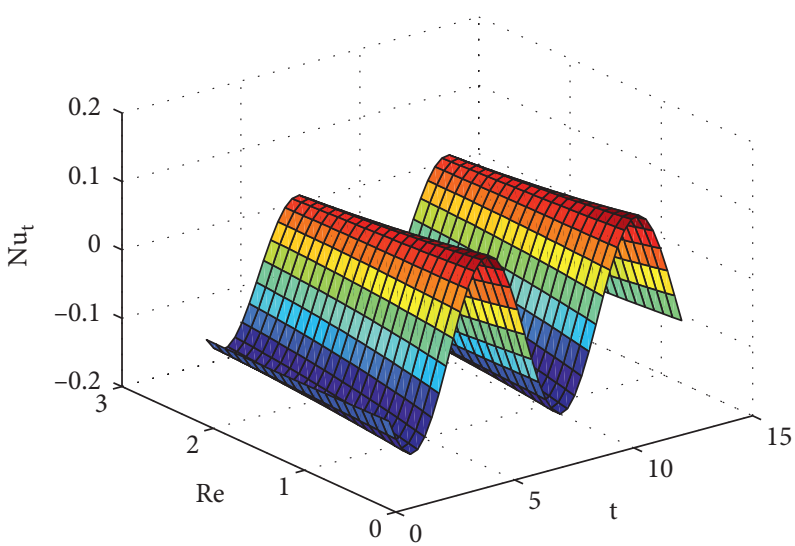

Figure 14: Effect of Re over time variation on the "unsteady Nusselt number distribution" for $\mathrm{Ec}=1, \mathrm{Rd}=2, \mathrm{Ha}=0.5$, and $\mathrm{Pr}=21$.

TABLE 2: Comparison of Nusselt number for channel with and without periodic body acceleration, when $\varepsilon=0.01, H=3, \beta=5, \mathrm{Ec}=1, \mathrm{Da}=0.2, \operatorname{Pr}=21$, and $t=\pi / 4$.

\begin{tabular}{|c|c|c|c|c|c|}
\hline \multirow[b]{2}{*}{ Parameter } & \multirow[b]{2}{*}{ Values } & \multicolumn{2}{|c|}{$\mathrm{Nu}=-(\partial \theta / \partial y)_{y=0}$} & \multicolumn{2}{|c|}{$\mathrm{Nu}=-(\partial \theta / \partial y)_{y=1}$} \\
\hline & & $\begin{array}{l}\text { Without body acceleration } \\
\qquad(G=0)\end{array}$ & $\begin{array}{l}\text { With body acceleration } \\
\qquad(G=1)\end{array}$ & $\begin{array}{l}\text { Without body acceleration } \\
\qquad(G=0)\end{array}$ & $\begin{array}{l}\text { With body acceleration } \\
\qquad(G=1)\end{array}$ \\
\hline \multirow{3}{*}{$\mathrm{Ha}$} & 0 & -5.0478 & -5.5719 & 6.2828 & 8.1745 \\
\hline & 1 & -4.7406 & -5.2344 & 8.7987 & 10.7217 \\
\hline & 2 & -3.9957 & -4.4158 & 15.0043 & 16.9792 \\
\hline \multirow{3}{*}{$\operatorname{Re}$} & 0 & -28.7997 & -31.2494 & 9.6086 & 10.7870 \\
\hline & 2 & -4.9676 & -5.4839 & 6.9407 & 8.8376 \\
\hline & 4 & -1.4012 & -1.5520 & -8.8930 & -7.7016 \\
\hline \multirow{3}{*}{ Q } & 0 & -4.8737 & -5.3805 & 4.0757 & 5.7305 \\
\hline & 0.5 & -4.9676 & -5.4839 & 6.9407 & 8.8376 \\
\hline & 1 & -5.0705 & -5.5971 & 10.4949 & 12.6975 \\
\hline \multirow{3}{*}{$\mathrm{Rd}$} & 1 & -5.3459 & -5.9066 & 13.2025 & 16.3828 \\
\hline & 2 & -4.9676 & -5.4839 & 6.9407 & 8.8376 \\
\hline & 3 & -4.6292 & -5.1053 & 4.0732 & 5.3677 \\
\hline
\end{tabular}

the Nusselt number $\mathrm{Nu}$ enhances at the lower plate for both the cases with the higher values of Re and Rd, while the inverse trend is noticed for larger value of $Q$. However, this behaviour is reversed at the upper plate for
$\operatorname{Re}, \mathrm{Rd}$, and $\mathrm{Q}$, while it increases for enlarging values of $\mathrm{Ha}$ at both the plates. It is also observed that with increasing periodic body acceleration $(G)$, the $\mathrm{Nu}$ decreases at the lower plate, while it increases at the upper 
plate. Therefore, the body acceleration reduces the heat conduction in lower temperature $\left(T_{0}\right)$, while it is enhanced at higher temperature $\left(T_{w}\right)$.

\section{Conclusion}

The present study addressed the influence of periodic body acceleration and thermal radiation on the MHD flow of Casson nanofluid through a porous channel. The pulsating flow is included in the flow problem. The analytical technique (perturbation) is utilized to compute the physical system. The considered problem is essential as the study related to periodic body acceleration in the porous channel has enormous applications in industrial and biological sectors. The generalized solutions have been found for the velocity and temperature distributions. Various graphs were depicted to emphasize vivid effects of various parameters, such as Reynolds number, frequency parameter, body acceleration parameter, Casson fluid parameter, radiation parameter, nanoparticle volume fraction, and the Darcy number:

(1) The fluid velocity increased for elevated values of frequency parameter, Casson fluid parameter, body acceleration parameter, nanoparticle volume fraction, and the Darcy number. However, the reverse effect was depicted for growing values of the Hartmann number.

(2) Diminishing behaviour of fluid flow is observed for diverse values of cross Reynolds number and the Prandtl number. Prandtl number has an essential role in the heat transfer process.

(3) The Nusselt number declines at the lower plate and increases at the upper plate with the rise in the nanoparticle volume fraction, Darcy number, and body acceleration parameter.

(4) The unsteady Nusselt number distribution is a periodic function of " $t$ " with period $2 \pi$ with its lowest values near $2 n \pi$ and higher values near $(2 n+1) \pi$ for all integer values of $n$.

(5) The body acceleration enhances the velocity at both plates. However, it reduces the heat conduction in lower temperature $\left(T_{0}\right)$, while it increases at higher temperature $\left(T_{w}\right)$.

\section{Appendix}

$$
\begin{aligned}
& \alpha_{1}=\frac{((1 / \beta)+1)}{\left(1-\varphi+\left(\varphi \rho_{n} / \rho_{f}\right)\right)(1-\varphi)^{2.5}} \\
& \alpha_{2}=\frac{1}{\left(1-\varphi+\left(\varphi \rho_{n} / \rho_{f}\right)\right)}\left(\mathrm{Ha}^{2}+\frac{1}{(1-\varphi)^{2.5} \mathrm{Da}}\right),
\end{aligned}
$$

$$
A_{3}=\frac{H^{2}+G \cos \psi}{\alpha_{2}}
$$$$
m_{1,2}=\frac{\operatorname{Re} \pm \sqrt{\operatorname{Re}^{2}+4 \alpha_{1} \alpha_{2}}}{2 \alpha_{1}}
$$$$
A_{1}=\frac{A_{3}\left(e^{m_{2}}-1\right)+1}{\left(e^{m_{1}}-e^{m_{2}}\right)} \text {, }
$$$$
A_{2}=\frac{A_{3}\left(e^{m_{1}}-1\right)+1}{\left(e^{m_{2}}-e^{m_{1}}\right)},
$$$$
\alpha_{3}=\frac{1}{\left(1-\varphi+\left(\varphi \rho_{n} / \rho_{f}\right)\right)}\left(\mathrm{Ha}^{2}+\frac{1}{(1-\varphi)^{2.5} \mathrm{Da}}\right)+i H^{2},
$$$$
A_{6}=\frac{H^{2}}{\alpha_{3}},
$$

$m_{3,4}=\frac{\operatorname{Re} \pm \sqrt{\operatorname{Re}^{2}+4 \alpha_{1} \alpha_{3}}}{2 \alpha_{1}}$

$A_{4}=\frac{A_{6}\left(e^{m_{4}}-1\right)+1}{\left(e^{m_{3}}-e^{m_{4}}\right)}$

$A_{5}=\frac{A_{6}\left(e^{m_{3}}-1\right)+1}{\left(e^{m_{4}}-e^{m_{3}}\right)}$,

$\alpha_{4}=\frac{1}{\left(1-\varphi+\left(\varphi \rho_{n} / \rho_{f}\right)\right)}\left(\mathrm{Ha}^{2}+\frac{1}{(1-\varphi)^{2.5} \mathrm{Da}}\right)+2 i H^{2}$,

$A_{9}=\frac{H^{2}}{\alpha_{4}}$,

$m_{5,6}=\frac{\operatorname{Re} \pm \sqrt{\operatorname{Re}^{2}+4 \alpha_{1} \alpha_{4}}}{2 \alpha_{1}}$,

$A_{7}=\frac{A_{9}\left(e^{m_{6}}-1\right)+1}{\left(e^{m_{5}}-e^{m_{6}}\right)}$,

$A_{8}=\frac{A_{9}\left(e^{m_{5}}-1\right)+1}{\left(e^{m_{6}}-e^{m_{5}}\right)}$,

$\beta_{1}=\frac{\left(\left(k_{n f} / k_{f}\right)+(4 / 3) \mathrm{Rd}\right)}{\operatorname{Pr}}$,

$\beta_{2}=\operatorname{Re}\left(1-\varphi+\frac{\varphi\left(\rho C_{p}\right)_{n}}{\left(\rho C_{p}\right)_{f}}\right)$

$m_{7,8}=\frac{\beta_{2} \pm \sqrt{\beta_{2}^{2}+4 \beta_{1} Q}}{2 \beta_{1}}$, 
14

Complexity

$$
\begin{aligned}
& \beta_{3}=\frac{(1+(1 / \beta))}{(1-\varphi)^{2.5}} \mathrm{Ec}, \\
& \beta_{4}=\mathrm{Ha}^{2} \mathrm{Ec}
\end{aligned}
$$

$$
\begin{aligned}
& B_{1}=-\frac{\beta_{3} m_{1}^{2} A_{1}^{2}+\beta_{4} A_{1}^{2}}{4 m_{1}^{2} \beta_{1}-2 m_{1} \beta_{2}+Q} \\
& B_{2}=-\frac{\beta_{3} m_{2}^{2} A_{2}^{2}+\beta_{4} A_{2}^{2}}{4 m_{2}^{2} \beta_{1}-2 m_{2} \beta_{2}+Q} \\
& B_{3}=\frac{2 \beta_{4} A_{1} A_{3}}{m_{1}^{2} \beta_{1}-m_{1} \beta_{2}+Q} \\
& B_{4}=\frac{2 \beta_{4} A_{2} A_{3}}{m_{2}^{2} \beta_{1}-m_{2} \beta_{2}+Q} \\
& B_{5}=-\frac{2 \beta_{3} m_{1} m_{2} A_{1} A_{2}+2 \beta_{4} A_{1} A_{2}}{\left(m_{1}+m_{2}\right)^{2} \beta_{1}-\left(m_{1}+m_{2}\right) \beta_{2}+Q}, \\
& B_{6}=\frac{\beta_{4} A_{3}^{2}}{Q} \\
& B_{7}=\frac{1}{\left(e^{m_{8}}-e^{m_{7}}\right)}\left(1+B_{1}\left(e^{m_{7}}-e^{2 m_{1}}\right)-B_{2}\left(e^{m_{7}}-e^{2 m_{2}}\right)\right. \\
& +B_{3}\left(e^{m_{7}}-e^{m_{1}}\right)+B_{4}\left(e^{m_{7}}-e^{m_{2}}\right) \\
& \left.+B_{5}\left(e^{m_{7}}-e^{\left(m_{1}+m_{2}\right)}\right)+B_{6}\left(e^{m_{7}}-1\right)\right), \\
& B_{8}=-\left(B_{6}+B_{7}+B_{5}+B_{3}+B_{4}+B_{2}+B_{1}\right) \text {, } \\
& \beta_{5}=Q-i\left(1-\varphi+\frac{\varphi\left(\rho C_{p}\right)_{n}}{\left(\rho C_{p}\right)_{f}}\right) H^{2} \\
& m_{9,10}=\frac{\beta_{2} \pm \sqrt{\beta_{2}^{2}+4 \beta_{1} \beta_{5}}}{2 \beta_{1}}, \\
& B_{9}=-\frac{2 \beta_{3} m_{1} m_{3} A_{1} A_{5}+2 \beta_{4} A_{1} A_{5}}{\left(m_{1}+m_{3}\right)^{2} \beta_{1}-\left(m_{1}+m_{3}\right) \beta_{2}+\beta_{5}}, \\
& B_{10}=-\frac{2 \beta_{3} m_{1} m_{4} A_{1} A_{4}+2 \beta_{4} A_{1} A_{4}}{\left(m_{1}+m_{4}\right)^{2} \beta_{1}-\left(m_{1}+m_{4}\right) \beta_{2}+\beta_{5}}, \\
& B_{11}=-\frac{2 \beta_{3} m_{2} m_{3} A_{2} A_{5}+2 \beta_{4} A_{2} A_{5}}{\left(m_{2}+m_{3}\right)^{2} \beta_{1}-\left(m_{2}+m_{3}\right) \beta_{2}+\beta_{5}}, \\
& B_{12}=-\frac{2 \beta_{3} m_{2} m_{4} A_{2} A_{4}+2 \beta_{4} A_{2} A_{4}}{\left(m_{2}+m_{4}\right)^{2} \beta_{1}-\left(m_{2}+m_{4}\right) \beta_{2}+\beta_{5}}, \\
& B_{13}=\frac{2 \beta_{4} A_{1} A_{6}}{m_{1}^{2} \beta_{1}-m_{1} \beta_{2}+\beta_{5}},
\end{aligned}
$$

$$
\begin{aligned}
& B_{14}=\frac{2 \beta_{4} A_{2} A_{6}}{m_{2}^{2} \beta_{1}-m_{2} \beta_{2}+\beta_{5}}, \\
& B_{15}=\frac{2 \beta_{4} A_{3} A_{5}}{m_{3}^{2} \beta_{1}-m_{3} \beta_{2}+\beta_{5}}, \\
& B_{16}=\frac{2 \beta_{4} A_{3} A_{4}}{m_{4}^{2} \beta_{1}-m_{4} \beta_{2}+\beta_{5}} \text {, } \\
& B_{17}=-\frac{2 \beta_{4} A_{3} A_{6}}{\beta_{5}}, \\
& B_{19}=\frac{1}{\left(e^{m_{10}}-e^{m_{9}}\right)}\left(B_{9}\left(e^{m_{9}}-e^{\left(m_{1}+m_{3}\right)}\right)\right. \\
& +B_{10}\left(e^{m_{9}}-e^{\left(m_{1}+m_{4}\right)}\right)-B_{11}\left(e^{m_{9}}-e^{\left(m_{2}+m_{3}\right)}\right) \\
& +B_{12}\left(e^{m_{9}}-e^{\left(m_{4}+m_{2}\right)}\right) \\
& +B_{13}\left(e^{m_{9}}-e^{m_{1}}\right) \\
& +B_{14}\left(e^{m_{9}}-e^{m_{2}}\right)+B_{15}\left(e^{m_{9}}-e^{m_{3}}\right)+B_{16}\left(e^{m_{9}}-e^{m_{4}}\right) \\
& +B_{17}\left(e^{m_{9}}-1\right) \text {, } \\
& B_{18}=-\left(B_{10}+B_{9}+B_{11}+B_{13}+B_{12}+B_{14}+B_{16}\right. \\
& \left.+B_{15}+B_{17}+B_{19}\right) \text {, } \\
& \beta_{6}=Q-2 i\left(1-\varphi+\frac{\varphi\left(\rho C_{p}\right)_{n}}{\left(\rho C_{p}\right)_{f}}\right) H^{2}, \\
& m_{11,12}=\frac{\beta_{2} \pm \sqrt{\beta_{2}^{2}+4 \beta_{1} \beta_{6}}}{2 \beta_{1}}, \\
& B_{20}=-\frac{\beta_{3} m_{3}^{2} A_{5}^{2}+\beta_{4} A_{5}^{2}}{m_{3}^{2} \beta_{1}-2 m_{3} \beta_{2}+\beta_{6}}, \\
& B_{21}=-\frac{\beta_{3} m_{4}^{2} A_{4}^{2}+\beta_{4} A_{4}^{2}}{m_{4}^{2} \beta_{1}-2 m_{4} \beta_{2}+\beta_{6}}, \\
& B_{22}=-\frac{2 \beta_{3} m_{3} m_{4} A_{4} A_{5}+2 \beta_{4} A_{4} A_{5}}{\left(m_{3}+m_{4}\right)^{2} \beta_{1}-\left(m_{3}+m_{4}\right) \beta_{2}+\beta_{6}}, \\
& B_{23}=-\frac{2 \beta_{3} m_{1} m_{3} A_{1} A_{5}}{\left(m_{1}+m_{3}\right)^{2} \beta_{1}-\left(m_{1}+m_{2}\right) \beta_{2}+\beta_{6}} \text {, } \\
& B_{24}=-\frac{2 \beta_{3} m_{1} m_{4} A_{1} A_{4}}{\left(m_{1}+m_{4}\right)^{2} \beta_{1}-\left(m_{1}+m_{4}\right) \beta_{2}+\beta_{6}} \text {, } \\
& B_{25}=-\frac{2 \beta_{3} m_{2} m_{3} A_{2} A_{5}}{\left(m_{2}+m_{3}\right)^{2} \beta_{1}-\left(m_{2}+m_{3}\right) \beta_{2}+\beta_{6}} \text {, }
\end{aligned}
$$




$$
\begin{aligned}
& B_{26}=-\frac{2 \beta_{3} m_{2} m_{4} A_{2} A_{4}}{\left(m_{2}+m_{4}\right)^{2} \beta_{1}-\left(m_{2}+m_{4}\right) \beta_{2}+\beta_{6}}, \\
& B_{27}=-\frac{2 \beta_{4} A_{1} A_{7}}{\left(m_{1}+m_{5}\right)^{2} \beta_{1}-\left(m_{1}+m_{5}\right) \beta_{2}+\beta_{6}}, \\
& B_{28}=-\frac{2 \beta_{4} A_{1} A_{8}}{\left(m_{1}+m_{6}\right)^{2} \beta_{1}-\left(m_{1}+m_{6}\right) \beta_{2}+\beta_{6}}, \\
& B_{29}=-\frac{2 \beta_{4} A_{2} A_{7}}{\left(m_{2}+m_{5}\right)^{2} \beta_{1}-\left(m_{2}+m_{5}\right) \beta_{2}+\beta_{6}}, \\
& B_{30}=-\frac{2 \beta_{4} A_{2} A_{8}}{\left(m_{2}+m_{6}\right)^{2} \beta_{1}-\left(m_{2}+m_{6}\right) \beta_{2}+\beta_{6}}, \\
& B_{31}=\frac{2 \beta_{4} A_{5} A_{6}}{m_{3}^{2} \beta_{1}-m_{3} \beta_{2}+\beta_{6}}, \\
& B_{32}=\frac{2 \beta_{4} A_{4} A_{6}}{m_{4}^{2} \beta_{1}-m_{4} \beta_{2}+\beta_{6}}, \\
& B_{33}=\frac{2 \beta_{4} A_{3} A_{7}}{m_{5}^{2} \beta_{1}-m_{5} \beta_{2}+\beta_{6}}, \\
& B_{34}=\frac{2 \beta_{4} A_{3} A_{8}}{m_{6}^{2} \beta_{1}-m_{6} \beta_{2}+\beta_{6}}, \\
& B_{35}=\frac{\beta_{4} A_{6}^{2}}{\beta_{6}}, \\
& B_{36}=\frac{1}{\left(e^{m_{11}}-e^{m_{12}}\right)}\left(B_{20}\left(e^{m_{11}}-e^{2 m_{3}}\right)+B_{21}\left(e^{m_{11}}-e^{2 m_{4}}\right)\right. \\
& +B_{22}\left(e^{m_{11}}-e^{\left(m_{4}+m_{3}\right)}\right)+B_{23}\left(e^{m_{11}}-e^{\left(m_{1}+m_{3}\right)}\right) \\
& +B_{24}\left(e^{m_{11}}-e^{\left(m_{1}+m_{4}\right)}\right)+B_{25}\left(e^{m_{11}}-e^{\left(m_{2}+m_{3}\right)}\right) \\
& +B_{26}\left(e^{m_{11}}-e^{\left(m_{4}+m_{2}\right)}\right)+B_{27}\left(e^{m_{11}}-e^{\left(m_{1}+m_{5}\right)}\right) \\
& +B_{28}\left(e^{m_{11}}-e^{\left(m_{1}+m_{6}\right)}\right) \\
& +B_{29}\left(e^{m_{11}}-e^{\left(m_{2}+m_{5}\right)}\right)+B_{30}\left(e^{m_{11}}-e^{\left(m_{6}+m_{2}\right)}\right) \\
& +B_{31}\left(e^{m_{11}}-e^{m_{3}}\right)+B_{32}\left(e^{m_{11}}-e^{m_{4}}\right)+B_{33}\left(e^{m_{11}}-e^{m_{5}}\right) \\
& +B_{34}\left(e^{m_{11}}-e^{m_{6}}\right)+B_{35}\left(e^{m_{11}}-1\right) \text {, } \\
& B_{37}=-\left(B_{21}+B_{22}+B_{28}+B_{26}+B_{29}+B_{27}+B_{31}+B_{34}\right. \\
& \left.+B_{32}+B_{30}+B_{36}+B_{25}+B_{33}+B_{23}+B_{24}+B_{35}+B_{20}\right) \text {. }
\end{aligned}
$$

\section{Nomencluature}


$n$ : Nanoparticle fraction

$n f$ : Nanofluid.

\section{Data Availability}

The raw data supporting the conclusions of this article are available from the corresponding author upon request.

\section{Conflicts of Interest}

The authors declare that they have no conflicts of interest.

\section{Acknowledgments}

The authors are grateful to Research and Development Wing, Live4Research, Tiruppur, Tamil Nadu, India, for the technical and financial support to this project work.

\section{References}

[1] M. Tamoor, M. Waqas, M. I. Khan, A. Alsaedi, and T. Hayat, "Magnetohydrodynamic flow of Casson fluid over a stretching cylinder," Results in Physics, vol. 7, pp. 498-502, 2017.

[2] P. B. A. Reddy, "Magnetohydrodynamic flow of a Casson fluid over an exponentially inclined permeable stretching surface with thermal radiation and chemical reaction," Ain Shams Engineering Journal, vol. 7, no. 2, pp. 593-602, 2016.

[3] S. Choi, "Enhancing thermal conductivity of fluids with nanoparticles," in Proceedings of the ASME International Mechanical Engineering Congress \& Exposition, pp. 99-105, San Francisco, CA, USA, January 1995.

[4] N. Sandeep, A. J. Chamkha, and I. L. Animasaun, "Numerical exploration of magnetohydrodynamic nanofluid flow suspended with magnetite nanoparticles," Journal of the Brazilian Society of Mechanical Sciences and Engineering, vol. 39, no. 2, pp. 3635-3644, 2017.

[5] K. S. Nisar, U. Khan, A. Zaib, I. Khan, and A. Morsy, "A novel study of radioactive flow involving micropolar nanoliquid from a shrinking/stretching curved surface including blood gold nanoparticles," The European Physical Journal Plus, vol. 135, no. 10, pp. 1-19, 2020.

[6] M. H. Abolbashari, N. Freidoonimehr, F. Nazari, and M. M. Rashidi, "Analytical modeling of entropy generation for Casson nano-fluid flow induced by a stretching surface," Advanced Powder Technology, vol. 231, no. 2, pp. 542-552, 2015.

[7] A. Tassaddiq, I. Khan, K. Sooppy Nisar, and J. Singh, "MHD flow of a generalized Casson fluid with Newtonian heating: a fractional model with Mittag-Leffler memory," Alexandria Engineering Journal, vol. 59, no. 5, pp. 3049-3059, 2020.

[8] Y. Menni, H. Ameur, and M. Inc, "Improvement of the performance of solar channels by using vortex generators and hydrogen fluid," Journal of Thermal Analysis and Calorimetry, 2020.

[9] Y. Menni, A. J. Chamkha, M. Ghazvini et al., "Enhancement of the turbulent convective heat transfer in channels through the baffling technique and oil/multiwalled carbon nanotube nanofluids," Numerical Heat Transfer Applications, vol. 79, no. 4, pp. 1-41, 2021.

[10] J. A. Khan, M. Mustafa, T. Hayat, M. A. Farooq, A. Alsaedi, and S. J. Liao, "On model for three-dimensional flow of nanofluid: an application to solar energy," Journal of Molecular Liquids, vol. 194, pp. 41-47, 2014.
[11] R. Maouedj, Y. Menni, M. Inc, Y.-M. Chu, H. Ameur, and G. Lorenzini, "Simulating the turbulent hydrothermal behavior of oil/MWCNT nanofluid in a Solar channel heat exchanger equipped with vortex generators," Computer Modeling in Engineering \& Sciences, vol. 126, no. 3, pp. 855-889, 2021.

[12] M. Irfan, M. A. Farooq, A. Mushtaq, and Z. H. Shamsi, "Unsteady MHD bionanofluid flow in a porous medium with thermal radiation near a stretching/shrinking sheet," Mathematical Problems in Engineering, vol. 2020, Article ID 8822999, 14 pages, 2020.

[13] N. Vellanki, K. Hemalatha, and G. V. R. Reddy, "Radiation and chemical reaction effects on MHD Casson fluid flow of a porous medium with suction/injection," International Journal of Mechanical Engineering and Technology, vol. 11, no. 2, pp. 99-116, 2020.

[14] J. A. Falede and S. O. Adensanya, "MHD oscillatory flow through porous channel saturated with porous media," Alexandria Engineering Journal, vol. 56, no. 1, pp. 147-152, 2017.

[15] K. Dwivedi, R. K. Khare, and A. Paul, "MHD flow through a vertical channel with porous medium," International Journal of Applied Engineering Research, vol. 13, pp. 1192311926, 2018.

[16] Y. Peng, A. S. Alsagri, M. Afrand, and R. Moradi, "A numerical simulation for magnetohydrodynamic nanofluid flow and heat transfer in rotating horizontal annulus with thermal radiation," RSC Advances, vol. 9, no. 39, pp. 22185-22197, 2019.

[17] A. Sharma and A. V. Dubewar, "MHD flow between two parallel plates under the influence of inclined magnetic field by finite difference method," International Journal of Innovative Technology and Exploring Engineering, vol. 8, no. 12, 2019.

[18] K. Loganathan, K. Mohana, M. Mohanraj, P. Sakthivel, and S. Rajan, "Impact of third-grade nanofluid flow across a convective surface in the presence of inclined Lorentz force: an approach to entropy optimization," Journal of Thermal Analysis and Calorimetry, vol. 144, no. 5, pp. 1935-1947, 2021.

[19] T. Anwar, P. Kumam, D. Baleanu, I. Khan, and P. Thounthong, "Radiative heat transfer enhancement in MHD porous channel flow of an Oldroyd-B fluid under generalized boundary conditions," Physica Scripta, vol. 95, no. 11, 2020.

[20] S. Pramanik, "Casson fluid flow and heat transfer past an exponentially porous stretching surface in presence of thermal radiation," Ain Shams Engineering Journal, vol. 5, no. 1, pp. 205-212, 2014.

[21] K. Loganathan, N. Alessa, K. Tamilvanan, and F. S. Alshammari, "Significances of Darcy-Forchheimer porous medium in third-grade nanofluid flow with entropy features," The European Physical Journal Special Topics, vol. 230, pp. 1293-1305, 2021.

[22] M. A. A. Hamad, M. J. Uddin, and A. I. M. Ismail, "Radiation effects on heat and mass transfer in MHD stagnation-point flow over a permeable flat plate with thermal convective surface boundary condition, temperature dependent viscosity and thermal conductivity," Nuclear Engineering and Design, vol. 242, pp. 194-200, 2012.

[23] K. Loganathan, G. Muhiuddin, A. M. Alanazi, F. S. Alshammari, B. M. Alqurashi, and S. Rajan, "Entropy optimization of third-grade nanofluid slip flow embedded in a porous sheet with zero mass flux and a non-Fourier heat flux model," Frontiers in Physics, vol. 8, p. 250. 
[24] M. G. Reddy, "Heat generation and thermal radiation effects over a stretching sheet in a micropolar fluid," International Scholarly Research notices, vol. 2012, Article ID 795814, 2012.

[25] A. Jamaludin, K. Naganthran, R. Nazar, and L. Pop, “Thermal radiation and MHD effects in the mixed convection flow of $\mathrm{Fe}_{3} \mathrm{O}_{4}$-water Ferrofluid towards a nonlinearly moving surface," Process, vol. 8, no. 1, p. 95, 2020.

[26] K. Loganathan and S. Rajan, "An entropy approach of Williamson nanofluid flow with Joule heating and zero nanoparticle mass flux," Journal of Thermal Analysis and Calorimetry, vol. 141, no. 6, pp. 2599-2612, 2020.

[27] M. Sheikholeslami, D. Domiri Ganji, M. Younus Javed, and R. Ellahi, "Effect of thermal radiation on magnetohydrodynamics nanofluid flow and heat transfer by means of two phase model," Journal of Magnetism and Magnetic Materials, vol. 374, pp. 36-43, 2015.

[28] K. Loganathan, N. Alessa, and S. Kayikci, "Heat transfer analysis of 3-D viscoelastic nanofluid flow over a convectively heated porous riga plate with Cattaneo-Christov double flux," Frontiers in Physics, vol. 9, Article ID 641645, 2021.

[29] S. Srinivas, C. K. Kumar, and A. S. Reddy, "Pulsating flow of Casson fluid in a porous channel with thermal radiation, chemical reaction and applied magnetic field," Nonlinear Analysis Modelling and Control, vol. 23, no. 2, pp. 213-233, 2018.

[30] C. K. Kumar, S. Srinivas, and A. S. Reddy, "MHD pulsating flow of Casson nanofluid in a vertical porous space with thermal radiation and joule heating," Journal of Mechanics, vol. 36, no. 4, pp. 1-15, 2020.

[31] B. Vishali and G. Sarojamma, "Effect of a periodic body acceleration on fluid flow through a catheterized artery," International Journal of Scintific and Innovative Mathematical Research, vol. 3, no. 7, pp. 26-35, 2015.

[32] P. Sulochana, "Unsteady MHD pulsatile flow of couple stress fluid under the influence of periodic body acceleration," Advances in Applied Science Research, vol. 5, no. 4, pp. 136143, 2014.

[33] G. M. Sobamowo, "Perturbation methods to analysis of thermal, fluid flow and dynamics behaviours of engineering systems," A Collection of Papers on Chaos Theory and its Applications, 2021, https://www.intechopen.com/chapters/ 75426.

[34] A. Kumar, "Perturbation technique to unsteady MHD periodic flow of viscous fluid through a planer channel," Journal of Engineering and Technology Research, vol. 2, no. 4, pp. 73-81, 2010.

[35] N. Thamaraikannan, S. Karthikeyan, and D. K. Chaudhary, "Significance of MHD radiative non-Newtonian nanofluid flow towards a porous channel: a framework of the Casson fluid model," Journal of Mathematics, vol. 2021, Article ID 9912239, 15 pages, 2021. 